ESAIM: COCV 26 (2020) 28

https://doi.org/10.1051/cocv/2019011
ESAIM: Control, Optimisation and Calculus of Variations

www.esaim-cocv.org

\title{
THRESHOLDING GRADIENT METHODS IN HILBERT SPACES: SUPPORT IDENTIFICATION AND LINEAR CONVERGENCE
}

\author{
Guillaume Garrigos $^{1, *}$, Lorenzo Rosasco ${ }^{2,3}$ and Silvia Villa ${ }^{4}$
}

\begin{abstract}
We study the $\ell^{1}$ regularized least squares optimization problem in a separable Hilbert space. We show that the iterative soft-thresholding algorithm (ISTA) converges linearly, without making any assumption on the linear operator into play or on the problem. The result is obtained combining two key concepts: the notion of extended support, a finite set containing the support, and the notion of conditioning over finite-dimensional sets. We prove that ISTA identifies the solution extended support after a finite number of iterations, and we derive linear convergence from the conditioning property, which is always satisfied for $\ell^{1}$ regularized least squares problems. Our analysis extends to the entire class of thresholding gradient algorithms, for which we provide a conceptually new proof of strong convergence, as well as convergence rates.
\end{abstract}

Mathematics Subject Classification. 49M29, 65J10, 65J15, 65J20, 65J22, 65K15, 90C25, $90 \mathrm{C} 46$.

Received December 3, 2017. Accepted March 6, 2019.

\section{INTRODUCTION}

Recent works show that, for many problems of interest, favorable geometry can greatly improve theoretical results with respect to more general, worst-case perspective $[1,5,16,20]$. In this paper, we follow this perspective to analyze the convergence properties of thresholding gradient methods in separable Hilbert spaces. Our starting point is the now classic iterative soft-thresholding algorithm (ISTA) to minimize the function

$$
f(x)=\|x\|_{1}+\frac{1}{2}\|A x-y\|^{2},
$$

defined by an operator $A$ on $\ell^{2}(\mathbb{N})$ and where $\|\cdot\|_{1}$ is the $\ell^{1}$ norm.

From the seminal work [11], it is known that ISTA converges strongly in $\ell^{2}(\mathbb{N})$. This result is generalized in [9] to a wider class of algorithms, the so-called thresholding gradient methods, noting that these are special instances of the Forward-Backward algorithm, where the proximal step reduces to a thresholding step onto an orthonormal basis (Sect. 2). Typically, strong convergence in Hilbert spaces is the consequence of a particular

Keywords and phrases: Forward-Backward method, support identification, conditioning, convergence rates.

1 CNRS, École Normale Supérieure (DMA), 75005 Paris, France.

${ }^{2}$ LCSL, Istituto Italiano di Tecnologia and Massachusetts Institute of Technology, Cambridge, MA 02139, USA.

3 DIBRIS, Università degli Studi di Genova, 16146 Genova, Italy.

4 Dipartimento di Matematica, Università degli Studi di Genova, 16146 Genova, Italy.

* Corresponding author: guillaume.garrigos@ens.fr 
structure of the considered problem. Classic examples being even functions, functions for which the set of minimizers has a nonempty interior, or strongly convex functions [32]. Further examples are uniformly convex functions, or functions presenting a favorable geometry around their minimizers, like conditioned functions or Lojasiewicz functions, see e.g. $[4,20]$. Whether the properties of ISTA, and more generally thresholding gradient methods, can be explained from this perspective is not apparent from the analysis in $[9,11]$.

Our first contribution is revisiting these results providing such an explanation: for these algorithms, the whole sequence of iterates is fully contained in a specific finite-dimensional subspace, ensuring automatically strong convergence. The key argument in our analysis is that after a finite number of iterations, the iterates identify the so called extended support of their limit. This set coincides with the active constraints at the solution of the dual problem, and reduces to the support, if some qualification condition is satisfied.

Going further, we tackle the question of convergence rates, providing a unifying treatment of finite- and infinite-dimensional settings. In finite dimensions, it is clear that if $A$ is injective, then $f$ becomes a strongly convex function, which guarantees a linear convergence rate. In [21], it is shown, still in a finite-dimensional setting, that the linear rates hold just assuming $A$ to be injective on the extended support of the problem. This result is generalized in [8] to a Hilbert space setting, assuming $A$ to be injective on any subspace of finite support. Linear convergence is also obtained by assuming the limit solution to satisfy some nondegeneracy condition $[8,26]$. In fact, it was shown recently in [6] that, in finite dimensions, no assumption at all is needed to guarantee linear rates. Using a key result in [25], the function $f$ was shown to be 2-conditioned on its sublevel sets, and 2-conditioning is sufficient for linear rates [2]. Our identification result, mentioned above, allows to easily bridge the gap between the finite- and infinite-dimensional settings. Indeed, we show that in any separable Hilbert space, linear rates of convergence always hold for the soft-thresholding gradient algorithm under no further assumptions. Once again, the key argument to obtain linear rates is the fact that the iterates generated by the algorithm identify, in finite time, a set on which we know the function to have a favorable geometry.

The paper is organized as follows. In Section 2, we describe our setting and introduce the thresholding gradient method. We introduce the notion of extended support in Section 3, in which we show that the thresholding gradient algorithm identifies this extended support after a finite number of iterations (Thm. 3.10). In Section 4, we present some consequences of this result on the convergence of the algorithm. We first derive in Section 4.1 the strong convergence of the iterates, together with a general framework to guarantee rates. We then specify our analysis to the function (1.1) in Section 4.2, and show the linear convergence of ISTA (Thm. 4.8). We also consider in Section 4.3 an elastic-net modification of (1.1), by adding an $\ell^{p}$ regularization term, and provide rates as well, depending on the value of $p \in] 1,+\infty[$.

\section{Thresholding GRADIENT MEthods}

Notation. We introduce some notation we will use throughout this paper. $\mathcal{N}$ is a subset of $\mathbb{N}, X$ is a separable Hilbert space endowed with the scalar product $\langle\cdot, \cdot\rangle$, and $\left(e_{k}\right)_{k \in \mathcal{N}}$ is an orthonormal basis of $X$. Given $x \in X$ and $k \in \mathcal{N}$ we set $x_{k}=\left\langle x, e_{k}\right\rangle$. The support of $x$ is $\operatorname{supp}(x)=\left\{k \in \mathcal{N} \mid x_{k} \neq 0\right\}$. Analogously, given $C \subset X$, $C_{k}=\left\{\left\langle x, e_{k}\right\rangle: x \in C\right\}$. Given $J \subset \mathbb{N}$, the subspace supported by $J$ is denoted by $X_{J}=\{x \in X \mid \operatorname{supp}(x) \subset J\}$ and the subset of finitely supported vectors by $c_{00}=\{x \in X: \operatorname{supp}(x)$ is finite $\}$. Given a collection of intervals $\mathcal{I}=\left\{I_{k}\right\}_{k \in \mathcal{N}}$ of the real line, with a slight abuse of notation, we define

$$
\mathbb{B}_{\infty, \mathcal{I}}=\bigoplus_{k \in \mathcal{N}} I_{k}=\left\{x \in X: x=\sum_{k \in \mathcal{N}} t_{k} e_{k}, \text { with } t_{k} \in I_{k} \text { for every } k \in \mathcal{N}\right\}
$$

Note that $\bigoplus_{k \in \mathcal{N}} I_{k}$ is a subspace of $X$. Therefore, the components of each element of $\bigoplus_{k \in \mathcal{N}} I_{k}$ must be square summable. The closed ball of center $x \in X$ and radius $\delta \in] 0,+\infty\left[\right.$ is denoted by $\mathbb{B}_{X}(x, \delta)$. Let $C \subset X$ be a closed convex set. Its indicator and support functions are denoted $\delta_{C}$ and $\sigma_{C}$, respectively, and the projection onto $C$ is $\operatorname{proj}_{C}$. Moreover, int $C$, bd $C, \operatorname{ri} C$, and qri $C$ will denote respectively the interior, the boundary, the relative interior, and the quasi relative interior of $C$ ([4], Sect. 6.2). The set of proper convex lower semi-continuous 
functions from $X$ to $\mathbb{R} \cup\{+\infty\}$ is denoted by $\Gamma_{0}(X)$. Let $f \in \Gamma_{0}(X)$ and let $\left.r \in\right] 0,+\infty[$. The sublevel set of $f$ is $S_{f}(r)=\{x \in X \mid f(x)-\inf f<r\}$. The proximity operator of $f$ is defined as

$$
(\forall \lambda \in] 0,+\infty[) \quad \operatorname{prox}_{\lambda f}(x)=\operatorname{argmin}\left\{y \in X \mid f(y)+\frac{1}{2 \lambda}\|y-x\|^{2}\right\} .
$$

Let $I \subset \mathbb{R}$ be a closed interval. Then, $\operatorname{prox}_{\sigma_{I}}=$ soft $_{I}$, where

$$
(\forall t \in \mathbb{R}) \quad \operatorname{soft}_{I}(t)= \begin{cases}t-\inf I & \text { if } t<\inf I \\ 0 & \text { if } t \in I \\ t-\sup I & \text { if } t>\sup I\end{cases}
$$

is the soft-thresholder corresponding to $I$.

Problem and main hypotheses. We consider the general optimization problem

$$
\min _{x \in X} f(x), \quad f=g+h,
$$

where typically $h$ will play the role of a smooth data fidelity term, and $g$ will be a nonsmooth sparsity promoting regularizer. More precisely, we will make all along this paper the following set of hypotheses:

$$
\left\{\begin{aligned}
h & \in \Gamma_{0}(X) \text { is bounded from below, } \\
h & \text { is differentiable, and } \nabla h \text { is } L \text {-Lipschitz continuous on } X, L \in] 0,+\infty[, \\
g & =\sum_{k \in \mathcal{N}} g_{k}\left(\left\langle\cdot, e_{k}\right\rangle\right), \text { with } g_{k}=\psi_{k}+\sigma_{I_{k}}, \text { where: } \\
& - \text { for all } k \in \mathcal{N}, I_{k} \text { is a proper closed interval of } \mathbb{R}, \text { and } \mathcal{I}=\left\{I_{k}\right\}_{k \in \mathcal{N}}, \\
& -\exists \omega>0 \text { such that, for all } k \in \mathcal{N}, \quad[-\omega, \omega] \subset I_{k}, \\
& - \text { for all } k \in \mathcal{N}, \psi_{k} \in \Gamma_{0}(\mathbb{R}) \text { is differentiable at } 0 \text { with } \psi_{k}(0)=0 \text { and } \psi_{k}^{\prime}(0)=0 .
\end{aligned}\right.
$$

As stated in the above assumption, in this paper we focus on a specific class of functions $g$. They are given by the sum of a positive function minimized at the origin and a generalized $\ell^{1}$ norm, namely:

$$
\psi=\sum_{k \in \mathcal{N}} \psi_{k}, \quad\|\cdot\|_{1, \mathcal{I}}=\sum_{k \in \mathcal{N}} \sigma_{I_{k}}
$$

In [9] the following characterization has been proved: the proximity operators of such functions $g$ are the monotone operators $T: X \rightarrow X$ such that for all $x \in X, T(x)=\left(T_{k}\left(x_{k}\right)\right)_{k \in \mathcal{N}}$, for some $T_{k}: \mathbb{R} \rightarrow \mathbb{R}$ which satisfies

$$
(\forall k \in \mathcal{N}) \quad T_{k}\left(x_{k}\right)=0 \Longleftrightarrow x_{k} \in I_{k}
$$

A few examples of such, so called, thresholding operators are shown in Figure 1, and a more in-depth analysis can be found in [9].

A well-known approach to approximate solutions of $(\mathrm{P})$ is the Forward-Backward algorithm [4]

$$
\left.x^{0} \in X, \quad \lambda \in\right] 0,2 L^{-1}\left[, \quad x^{n+1}=\operatorname{prox}_{\lambda g}\left(x^{n}-\lambda \nabla h\left(x^{n}\right)\right) .\right.
$$



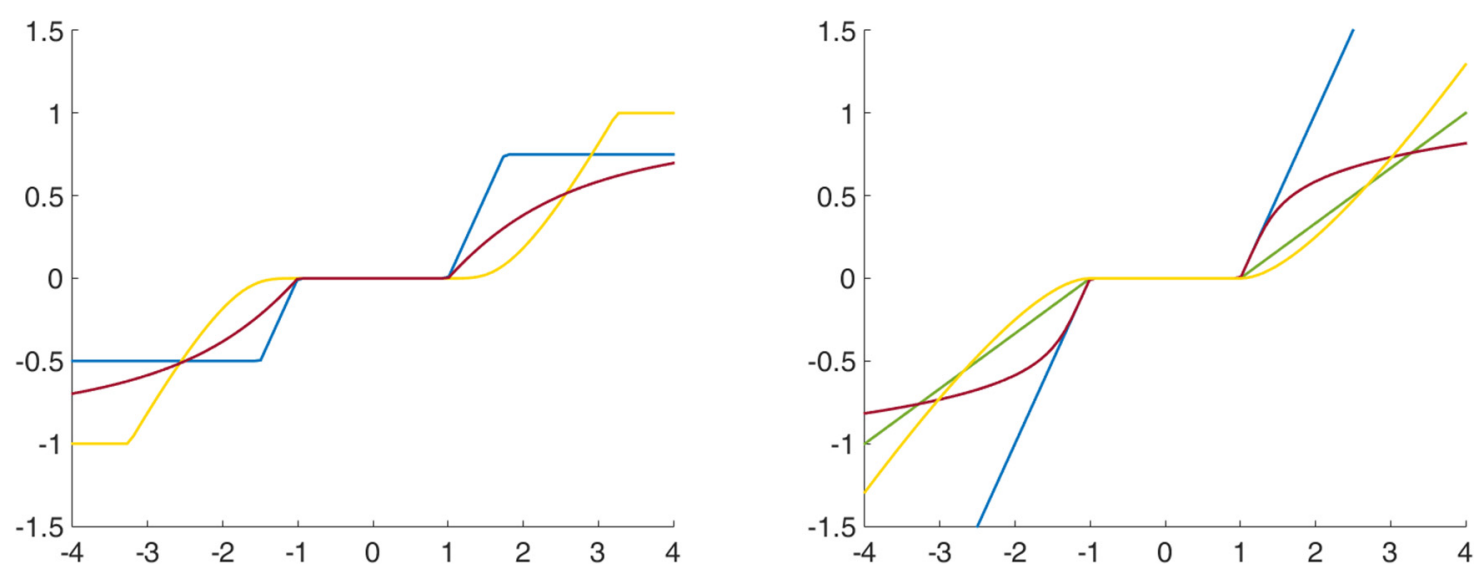

Figure 1. Some examples of thresholding proximal operators in $\mathbb{R}$. On the left: $|\cdot|=\sigma_{[-1,1]}$ (blue),$|\cdot|+|\cdot|^{1.5}$ (yellow),$|\cdot|+|\cdot|^{2}$ (green), and $|\cdot|+|\cdot|^{6}$ (red). On the right: $|\cdot|+\delta_{[-0.5,0.75]}$ (blue), $|\cdot|+|\cdot|^{1.5}+\delta_{[-1,1]}$ (yellow), and $|\cdot|-\ln (1-|\cdot|)+\delta_{-1,1[}($ red). Observe that here the range of $\operatorname{prox}_{g}$ is equal to the domain of $\partial \psi$.

In our setting, (FB) is well-defined and specializes to a thresholding gradient method, since $\operatorname{prox}_{\lambda g}$ is a thresholding operator. The proposition below gathers some basic properties of $g$ and $f$ following from assumption $(\mathrm{H})$.

\section{Proposition 2.1. The following hold.}

(i) $\|\cdot\|_{1, \mathcal{I}}$ is the support function of $\mathbb{B}_{\infty, \mathcal{I}}=\bigoplus_{k \in \mathcal{N}} I_{k}$,

(ii) $\operatorname{dom} \partial\|\cdot\|_{1, \mathcal{I}}=c_{00}$,

(iii) $g \in \Gamma_{0}(X)$ and it is coercive,

(iv) $f$ is bounded from below and $\operatorname{argmin} f$ is nonempty,

(v) the dual problem

$$
\min _{u \in X} g^{*}(u)+h^{*}(-u)
$$

admits a unique solution $\bar{u} \in X$, and for all $\bar{x} \in \operatorname{argmin} f, \bar{u}=-\nabla h(\bar{x})$.

(vi) for all $x \in X$ and all $\lambda>0$, the proximal operator of $g$ can be expressed as

$$
\operatorname{prox}_{\lambda g}(x)=\sum_{k \in \mathcal{N}} \operatorname{prox}_{\lambda \psi_{k}}\left(\operatorname{soft}_{\lambda I_{k}}\left(x_{k}\right)\right) e_{k}
$$

Proof. (i): see Proposition A.5(ii).

(ii): see Proposition A.5(iii).

(iii): see Proposition A.5(ii).

(iv): it is a consequence of the coercivity of $g$ and the fact that both $h$ and $g$ are bounded from below.

(v): the smoothness of $h$ implies the strong convexity of $h^{*}$, and the existence and uniqueness of $\bar{u}$, see ([4], Thms. 15.13 and 18.15). The equality $\bar{u}=-\nabla h(\bar{x})$ follows from ([4], Prop. 26.1(iv)(b)).

(vi): it follows from A.5(iv) together with ([9], Prop. 3.6). 


\section{EXTENDED SUPPORT AND FINITE IDENTIFICATION}

\subsection{Definition and basic properties}

We introduce the notion of extended support of a vector and prove some basic properties of the support of solutions of problem $(\mathrm{P})$.

Definition 3.1. Let $x \in X$. The extended support of $x$ is

$$
\operatorname{esupp}(x)=\operatorname{supp}(x) \cup\left\{k \in \mathcal{N} \mid-\nabla h(x)_{k} \in \operatorname{bd} I_{k}\right\}
$$

It is worth noting that the notion of extended support depends on the problem $(\mathrm{P})$, since its definition involves $h$ (see Rem. 3.4 for more details). It appears without a name in [21], and also in $[14,15,17]$ for regularized least squares problems. Below we gather some results about the support and the extended support.

Proposition 3.2. Let $x \in \operatorname{dom} \partial f$, then $\operatorname{supp}(x)$ and $\operatorname{esupp}(x)$ are finite.

Proof. Let $x \in \operatorname{dom} \partial f=\operatorname{dom} \partial g$, and let us start by verifying that $\operatorname{supp}(x)$ is finite. Let $x^{*} \in \partial g(x)$, and define $y=x+x^{*}$. By definition, $y \in x+\partial g(x)$, so $x=(I d+\partial g)^{-1}(y)=\operatorname{prox}_{g}(y)$. We deduce from this and Proposition 2.1(vi) that for all $k \in \operatorname{supp}(x), \operatorname{prox}_{\psi_{k}} \circ \operatorname{soft}_{I_{k}}\left(y_{k}\right) \neq 0$. Lemma A.4 and the definition of $\operatorname{soft}_{I_{k}}$ imply that $y_{k} \notin I_{k}$, and in particular that $\left|y_{k}\right| \geq \omega$ for all $k \in \operatorname{supp}(x)$. Then we derive that

$$
|\operatorname{supp}(x)|=\omega^{-2} \sum_{k \in \operatorname{supp}(x)} \omega^{2} \leq \omega^{-2} \sum_{k \in \operatorname{supp}(x)}\left|y_{k}\right|^{2} \leq \omega^{-2}\|y\|^{2}<+\infty
$$

Next, we have to verify that $J=\left\{k \in \mathcal{N} \mid-\nabla h(x)_{k} \in \operatorname{bd} I_{k}\right\}$ is finite. If $\mathcal{N}$ is finite, this is trivial. Otherwise, we observe that $\left(\nabla h(x)_{k}\right)_{k \in \mathcal{N}} \in \ell^{2}(\mathcal{N})$, which implies that $\nabla h(x)_{k}$ tends to 0 when $k \rightarrow+\infty$ in $\mathcal{N}$. Since $[-\omega, \omega] \subset I_{k}$, we deduce that $J$ must be finite.

The following proposition clarifies the relationship between the support and the extended support for minimizers.

Proposition 3.3. Let $\bar{x} \in \operatorname{argmin} f$.

(i) If $0 \in \operatorname{qri} \partial f(\bar{x})$ then $\operatorname{esupp}(\bar{x})=\operatorname{supp}(\bar{x})$.

Assume that $\psi_{k}$ is differentiable on dom $\partial \psi_{k}$, for all $k \in \mathcal{N}$. Then

(ii) $\operatorname{esupp}(\bar{x})=\operatorname{supp}(\bar{x}) \Leftrightarrow 0 \in \operatorname{qri} \partial f(\bar{x})$.

Assume moreover that $\psi \equiv 0$. Then

(iii) $\operatorname{esupp}(\bar{x})=\left\{k \in \mathcal{N} \mid-\nabla h(\bar{x})_{k} \in\right.$ bd $\left.I_{k}\right\}$.

(iv) There exists $J \subset \mathcal{N}$ such that $J=\operatorname{esupp}(\bar{x})$ for every $\bar{x} \in \operatorname{argmin} f$.

(v) $\operatorname{esupp}(\bar{x})=\cup\{\operatorname{supp}(x) \mid x \in \operatorname{argmin} f\} \Leftrightarrow(\exists x \in \operatorname{argmin} f) 0 \in \operatorname{qri} \partial f(x)$.

Proof. Since $\bar{x} \in \operatorname{argmin} f \subset$ dom $\partial g$, it follows from Proposition 3.2 that $\operatorname{supp}(\bar{x})$ is finite. Moreau-Rockafellar's sum rule ([31], Thm. 3.30), Proposition A.5(iii), Proposition A.1(i) then yield

$$
\partial f(\bar{x})_{k}=\nabla h(\bar{x})_{k}+ \begin{cases}\partial \psi_{k}\left(\bar{x}_{k}\right)+\partial \sigma_{I_{k}}\left(\bar{x}_{k}\right) & \text { if } k \in \operatorname{supp}(\bar{x}) \\ I_{k} & \text { if } k \notin \operatorname{supp}(\bar{x})\end{cases}
$$


Since $\operatorname{supp}(\bar{x})$ is finite and $\partial \psi_{k}\left(\bar{x}_{k}\right)+\partial \sigma_{I_{k}}\left(\bar{x}_{k}\right)$ is a closed interval of $\mathbb{R}$, Proposition A.3 and Proposition A.1(iii) imply

$$
(\forall k \in \mathcal{N}) \quad(\operatorname{qri} \partial f(\bar{x}))_{k}=\nabla h(\bar{x})_{k}+ \begin{cases}\operatorname{ri}\left(\partial \psi_{k}\left(\bar{x}_{k}\right)+\partial \sigma_{I_{k}}\left(\bar{x}_{k}\right)\right) & \text { if } k \in \operatorname{supp}(\bar{x}) \\ \operatorname{int} I_{k} & \text { if } k \notin \operatorname{supp}(\bar{x}) .\end{cases}
$$

(i): observe that

$$
\begin{aligned}
0 \in \operatorname{qri} \partial f(\bar{x}) & \Rightarrow(\forall k \notin \operatorname{supp}(\bar{x})) \quad-\nabla h(\bar{x})_{k} \in \operatorname{int} I_{k} \\
& \Leftrightarrow\left\{k \in \mathcal{N} \mid x_{k}=0 \text { and }-\nabla h(\bar{x})_{k} \in \operatorname{bd} I_{k}\right\}=\emptyset \\
& \Leftrightarrow \operatorname{esupp}(\bar{x})=\operatorname{supp}(\bar{x}) .
\end{aligned}
$$

(ii): note that from $0 \in \partial f(\bar{x})$ and (3.1), we have $-\nabla h(\bar{x})_{k} \in \partial \psi_{k}\left(\bar{x}_{k}\right)+\partial \sigma_{I_{k}}\left(\bar{x}_{k}\right)$ for all $k \in \operatorname{supp}(\bar{x})$. But both $\psi_{k}$ and $\sigma_{I_{k}}$ are differentiable at $\bar{x}_{k} \neq 0$, so for all $k \in \operatorname{supp}(\bar{x})$, qri $\left(\partial \psi_{k}\left(\bar{x}_{k}\right)+\partial \sigma_{I_{k}}\left(\bar{x}_{k}\right)\right)=\partial \psi_{k}\left(\bar{x}_{k}\right)+\partial \sigma_{I_{k}}\left(\bar{x}_{k}\right)$ holds. So, we deduce from (3.3) that item (ii) holds.

(iii): observe that, via (3.1) and Proposition A.1(ii), for all $k \in \operatorname{supp}(\bar{x}),-\nabla h(\bar{x})_{k} \in$ bd $I_{k}$, meaning that indeed $\operatorname{esupp}(\bar{x})=\left\{k \in \mathbb{N} \mid-\nabla h(\bar{x})_{k} \in\right.$ bd $\left.I_{k}\right\}$.

(iv): it follows from the uniqueness of $\nabla h(\bar{x})$ (see Prop. 2.1(v)) and item (iii).

(v): if there is some $x \in \operatorname{argmin} f$ such that $0 \in$ qri $\partial f(x)$, we derive from (ii) and (iv) that $\operatorname{esupp}(\bar{x})=\operatorname{supp}(x)$. So, the inclusion $\operatorname{esupp}(\bar{x}) \subset \cup\left\{\operatorname{supp}\left(x^{\prime}\right) \mid x^{\prime} \in \operatorname{argmin} f\right\}$ holds. For the reverse inclusion, note that Definition 3.1 and (iv) imply that $\operatorname{supp}(x) \subset \operatorname{esupp}(x)=\operatorname{esupp}(\bar{x})$ for all $x \in \operatorname{argmin} f$. For the reverse implication, assume that $\operatorname{esupp}(\bar{x})=\cup\{\operatorname{supp}(x) \mid x \in \operatorname{argmin} f\}$ holds, and use the fact that $\operatorname{esupp}(\bar{x})$ is finite to apply Lemma A.9, and obtain some $x \in \operatorname{argmin} f$ such that $\operatorname{supp}(x)=\operatorname{esupp}(\bar{x})$. We then conclude that $0 \in$ qri $\partial f(x)$ using (iv) and (ii).

Remark 3.4 (Extended support and active constraints). Assume that $\psi=0$. Since $g^{*}$ is the indicator function of $\mathbb{B}_{\infty, \mathcal{I}}$, in this case, the dual problem (D) introduced in Proposition 2.1(v) can be rewritten as

$$
\min _{\substack{u \in X \\(\forall k \in \mathcal{N}) u_{k} \in I_{k}}} h^{*}(-u)
$$

This problem admits a unique solution $\bar{u} \in \mathbb{B}_{\infty, \mathcal{I}}$, and the set of active constraints at $\bar{u}$ is

$$
\left\{k \in \mathcal{N} \mid \bar{u}_{k} \in \operatorname{bd} I_{k}\right\} .
$$

Since $\bar{u}=-\nabla h(\bar{x})$ for any $\bar{x} \in \operatorname{argmin} f$ by Proposition 2.1(v), Proposition 3.3(iii) implies that the extended support for the solutions of $(\mathrm{P})$ is in that case nothing but the set of active constraints for the solution of $\left(\mathrm{D}^{\prime}\right)$.

Remark 3.5 (Maximal support and interior solution). Consider the following (weak) qualification condition:

$$
(\exists \bar{x} \in \operatorname{argmin} f) 0 \in \operatorname{qri} \partial f(\bar{x}) .
$$

If $\psi=0$ and (w-CQ) holds then, thanks to Lemma A.9, we see that the extended support is exactly the maximal support to be found among the solutions. For instance, if $h$ is the least squares loss on a finite-dimensional space, it can be shown that the solutions having a maximal support are the ones belonging to the relative interior of the solution set ([3], Thm. 2). However, there are problems for which (w-CQ) does not hold. In such cases Proposition 3.3.(v) shows that the extended support will be strictly larger than this maximal support. Both cases are illustrated in the examples below. 

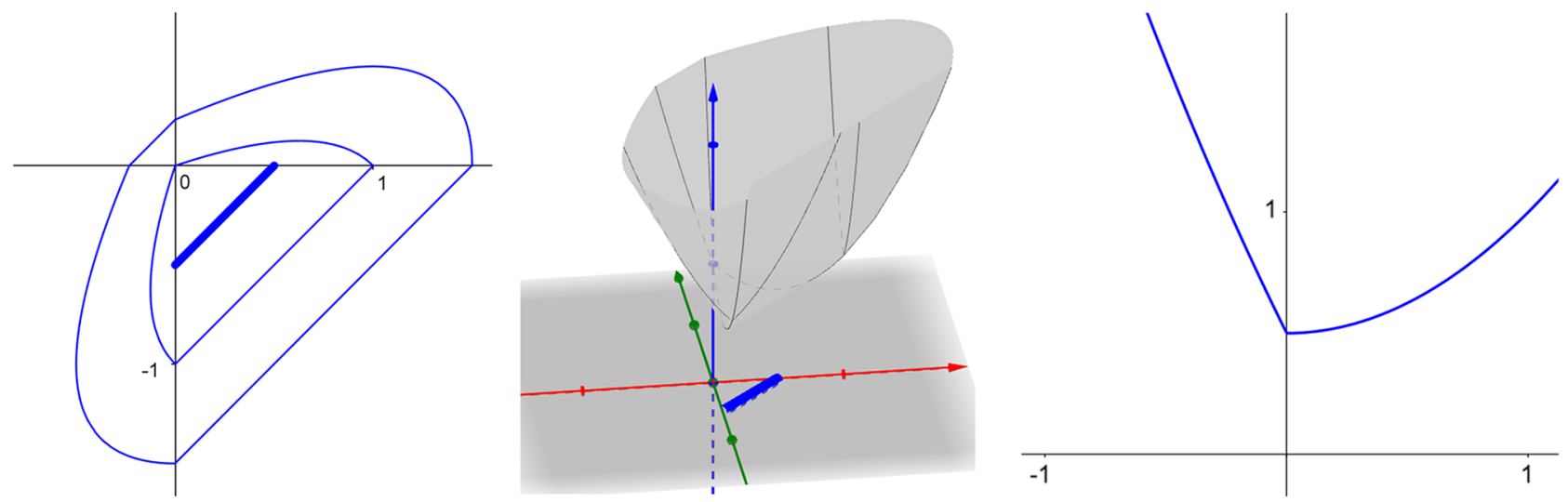

Figure 2. Left and center: respectively level sets and graph of $f$ in Example 3.6, with argmin $f$ in thick. Right: graph of $f$ in Example 3.7.

Example 3.6. Let $g: \mathbb{R}^{2} \rightarrow \mathbb{R}: x \mapsto\|x\|_{1}$ and $h: \mathbb{R}^{2} \rightarrow \mathbb{R}: x \mapsto\left(x_{1}-x_{2}-1\right)^{2}$. In this case, $\operatorname{argmin} f=\left[\bar{x}^{1}, \bar{x}^{2}\right]$, where $\bar{x}^{1}=(0.5,0)$ and $\bar{x}^{2}=(0,-0.5)$, as can be seen in Figure 2. The solutions $\left.\bar{x} \in\right] \bar{x}^{1}, \bar{x}^{2}$ [ are the ones having the maximal support, since $\operatorname{supp}(\bar{x})=\{1,2\}$, and they also satisfy ${ }^{1} 0 \in \operatorname{ri} \partial f(\bar{x})$. Instead, on the relative boundary of $\operatorname{argmin} f$ we have $\operatorname{supp}\left(\bar{x}^{i}\right)=\{i\}$ and $0 \notin \operatorname{ri} \partial f\left(\bar{x}^{i}\right)$ for $i \in\{1,2\}$. This example is a one for which the extended support is the maximal support among the solutions.

Example 3.7. Let $g: \mathbb{R} \rightarrow \mathbb{R}: x \mapsto|x|$ and $h: \mathbb{R} \rightarrow \mathbb{R}: x \mapsto(x-1)^{2} / 2$. Then $\operatorname{argmin} f=\{\bar{x}\}$, with $\bar{x}=0$, as can be seen in Figure 2. The support of $\bar{x}$ is empty, and $0 \notin \operatorname{ri} \partial f(\bar{x})=]-2,0[$. In this case, condition (w-CQ) does not hold. This can also be seen from the dual problem $\min _{u \in[-1,1]} u^{2} / 2-u$, whose unique constraint is active at the solution $\bar{u}=-\nabla h(\bar{x})=1$, meaning that $\operatorname{esupp}(\bar{x})=\{1\}$ is strictly larger than $\operatorname{supp}(\bar{x})$.

\subsection{Finite identification}

A sparse solution $\bar{x}$ of problem (P) is usually approximated by means of an iterative procedure $\left(x^{n}\right)_{n \in \mathbb{N}}$. To obtain an interpretable approximation, a crucial property is that, after a finite number of iterations, the support of $x^{n}$ stabilizes and is included in the support of $\bar{x}$. In that case, we say that the sequence $\left(x^{n}\right)_{n \in \mathbb{N}}$ identifies $\operatorname{supp}(\bar{x})$. The support identification property has been the subject of active research in the last years $[15,17,18,21,26]$, and roughly speaking, in finite dimensions it is known that support identification holds whenever $\bar{x}$ satisfies the qualification condition

$$
0 \in \operatorname{qri} \partial f(\bar{x}) .
$$

More precisely, in ([27], Prop. 3.6), the finite-dimensional case is studied, and the proof relies on (QC) to hold. This qualification condition ensures that for a large class of functions $g$ the support of the sequence becomes constant after a large enough number of iterations, and the proof is based on this property. But the qualification condition is not always satisfied by the points in $\operatorname{argmin} f$, as it is illustrated in Examples 3.6 and 3.7. In [21], the case $g(x)=\|x\|_{1}$ is studied in finite dimensions and it is shown that the extended support of $\bar{x}$ is identified even if the qualification condition does not hold. Thus, the qualification condition $0 \in \operatorname{qri} \partial f(\bar{x})$ is only used to ensure that the extended support coincides with the support (see Prop. 3.3). In this section, we extend these ideas to the setting of thresholding gradient methods in separable Hilbert spaces, and we show in Theorem 3.10 that indeed the extended support is always identified after a finite number of iterations. For this, we need to introduce a quantity, which measures the stability of the dual problem (D).

\footnotetext{
${ }^{\mathrm{I}}$ Note that the quasi relative interior coincides with the relative interior in finite dimensions ([4], Fact 6.14(i)).
} 
Definition 3.8. We define the function $\rho: X \longrightarrow \mathbb{R}$ as follows:

$$
(\forall u \in X) \quad \rho(u)=\inf _{u_{k} \in \operatorname{int} I_{k}} \operatorname{dist}\left(u_{k}, \text { bd } I_{k}\right) .
$$

Also, given any $\bar{x} \in \operatorname{argmin} f$, we define $\rho_{\text {sol }}=\rho(-\nabla h(\bar{x}))$.

Thanks to Proposition 2.1(v), we know that $\rho_{\text {sol }}$ is well-defined. Moreover, it satisfies $\rho_{\text {sol }}>0$, according to the following lemma (whose proof is postponed to the Annex, see Prop. A.2):

Lemma 3.9. For all $u \in X$ with $\operatorname{supp}(u) \neq X, \quad \rho(u) \in] 0,+\infty[$.

Note that the condition $\operatorname{supp}(u) \neq X$ is always satisfied in the infinite-dimensional setting.

Theorem 3.10 (Finite identification of the extended support). Let $\left(x^{n}\right)_{n \in \mathbb{N}}$ be generated by the ForwardBackward algorithm (FB), and let $\bar{x}$ be any minimizer of $f$. Then, the number of iterations for which the support of $x^{n}$ is not included in $\operatorname{esupp}(\bar{x})$ is finite, and cannot exceed $\rho_{\mathrm{sol}}^{-2} \lambda^{-2}\left\|x^{0}-\bar{x}\right\|^{2}$.

Remark 3.11 (Comparison with other identification results). Our result extends the bound obtained in a finitedimensional setting in ([21], Thm. 4.5). This bound can be improved by making additional assumptions on the problem. Still in finite dimensions, and assuming that the limit of the generated sequence $\bar{x}$ satisfies (QC), the authors in ([27], Prop. 3.6) improve this bound by replacing $\rho_{\text {sol }}$ with a tighter constant. While this paper was under review, the preprint [30] appeared online, also studying finite identification results in finite dimensions, assuming strong convexity of the objective function and a qualification condition. Under these stronger assumptions, which imply uniqueness of the solution and linear convergence of the thresholding gradient algorithm, it is possible to show that the algorithm faster identifies the support. The proof in this case relies on completely different arguments, which cannot be extended to the general convex case.

Remark 3.12 (Sharpness of the outer bound on the support). Let us assume for simplicity that $\psi=0$, meaning that the extended support is the same for all solutions $\bar{x} \in \operatorname{argmin} f$ (see Prop. 3.3.(iv)). We thus note here their extended support $\operatorname{esupp}_{\text {sol }}$ instead of $\operatorname{esupp}(\bar{x})$. In Theorem 3.10 we showed that

$$
\left(\forall x^{0} \in X\right)\left(\exists n_{0} \in \mathbb{N}\right)\left(\forall n \geq n_{0}\right) \quad \operatorname{supp}\left(x^{n}\right) \subset \operatorname{esupp}_{\text {sol }} .
$$

How sharp is this outer bound? More precisely, is there a problem, and a proper subset $J \varsubsetneqq \operatorname{esupp}_{\text {sol }}$ depending only on the problem, such that

$$
\left(\forall x^{0} \in X\right)\left(\exists n_{0} \in \mathbb{N}\right)\left(\forall n \geq n_{0}\right) \quad \operatorname{supp}\left(x^{n}\right) \subset J ?
$$

To answer this question, it is worth considering two cases. If (w-CQ) holds, it is easy to see that such $J$ cannot exists. Indeed, (w-CQ) implies the existence of some $\bar{x} \in \operatorname{argmin} f$ for which $0 \in \operatorname{qri} \partial f(\bar{x})$ holds. Starting from $x^{0}=\bar{x}$, it is easy to see that the generated sequence will be constant $x^{n}=\bar{x}$, and Proposition 3.3.(i) shows that $\operatorname{supp}\left(x^{n}\right)=\operatorname{esupp}_{\mathrm{sol}}$. If instead (w-CQ) fails to be satisfied, the question remains open. Observe that Example 3.7 cannot be used as a counter-example here, because if we start with $\left.x^{0} \in\right] 0,+\infty[$ and take a stepsize $\lambda \in] 0,1[$, then the iterates of $(\mathrm{FB})$ verify $x^{n+1}=(1-\lambda) x^{n}$, meaning that for all $n \in \mathbb{N}$, $\operatorname{supp}\left(x^{n}\right)=\{1\}=\operatorname{esupp}_{\text {sol }}$. This implies that for this specific problem, a strictly smaller $J$ cannot exists. But this says nothing about other problems for which (w-CQ) fails.

Proof of Theorem 3.10. Let $\bar{x} \in \operatorname{argmin} f$, and let $E=X_{\operatorname{esupp}(\bar{x})}$ be the finite-dimensional subspace of $X$ supported by $\operatorname{esupp}(\bar{x})$. First define the "gradient step" operator

$$
T_{\lambda h}=\mathrm{Id}-\lambda \nabla h,
$$


so that the Forward-Backward iteration can be rewritten as $x^{n+1}=\operatorname{prox}_{\lambda g}\left(T_{\lambda h}\left(x^{n}\right)\right)$. Proposition 2.1(vi) implies that for all $k \in \mathcal{N}$ and all $n \in \mathbb{N}^{*}$,

$$
x_{k}^{n}=\operatorname{prox}_{\lambda \psi_{k}} \circ \operatorname{soft}_{\lambda I_{k}}\left(T_{\lambda h}\left(x^{n-1}\right)_{k}\right) .
$$

Since $\bar{x}$ is a fixed point for the Forward-Backward iteration ([4], Prop. 26.1(iv)), we also have

$$
(\forall k \in \mathcal{N}) \quad \bar{x}_{k}=\operatorname{prox}_{\lambda \psi_{k}} \circ \operatorname{soft}_{\lambda I_{k}}\left(T_{\lambda h}(\bar{x})_{k}\right) .
$$

Using the fact that $\operatorname{prox}_{\lambda \psi_{k}}$ is nonexpansive, and that soft $_{\lambda I_{k}}$ is firmly non-expansive ([4], Prop. 12.28) for every $k \in \mathcal{N}$, we derive

$$
\begin{aligned}
\left\|x^{n}-\bar{x}\right\|^{2} & =\sum_{k \in \mathcal{N}}\left|x_{k}^{n}-\bar{x}_{k}\right|^{2} \leq \sum_{k \in \mathcal{N}}\left|\operatorname{soft}_{\lambda I_{k}}\left(T_{\lambda h}\left(x^{n-1}\right)_{k}\right)-\operatorname{soft}_{\lambda I_{k}}\left(T_{\lambda h}(\bar{x})_{k}\right)\right|^{2} \\
& \leq \sum_{k \in \mathcal{N}}\left|T_{\lambda h}\left(x^{n-1}\right)_{k}-T_{\lambda h}(\bar{x})_{k}\right|^{2}-\left|\left(\mathrm{Id}-\operatorname{soft}_{\lambda I_{k}}\right)\left(T_{\lambda h}\left(x^{n-1}\right)_{k}\right)-\left(\mathrm{Id}-\operatorname{soft}_{\lambda I_{k}}\right)\left(T_{\lambda h}(\bar{x})_{k}\right)\right|^{2} \\
& \leq\left\|T_{\lambda h}\left(x^{n-1}\right)-T_{\lambda h}(\bar{x})\right\|^{2}-\sum_{k \in \mathcal{N}} \sigma_{n, k}^{2},
\end{aligned}
$$

where

$$
(\forall k \in \mathcal{N})\left(\forall n \in \mathbb{N}^{*}\right) \quad \sigma_{n, k}:=\left|\left(\operatorname{Id}-\operatorname{soft}_{\lambda I_{k}}\right)\left(T_{\lambda h}\left(x^{n-1}\right)_{k}\right)-\left(\operatorname{Id}-\operatorname{soft}_{\lambda I_{k}}\right)\left(T_{\lambda h}(\bar{x})_{k}\right)\right| .
$$

Moreover, the gradient step operator $T_{\lambda h}$ is non-expansive since $\left.\lambda \in\right] 0,2 L^{-1}[$ (see e.g. [24], Lem. 3.2), and the $\sigma_{n, k}$ are positive, so we end up with

$$
\left(\forall n \in \mathbb{N}^{*}\right)(\forall k \in \mathcal{N}) \quad\left\|x^{n}-\bar{x}\right\|^{2} \leq\left\|x^{n-1}-\bar{x}\right\|^{2}-\sigma_{n, k}^{2} .
$$

The key point of the proof is to get for some $k \in \mathcal{N}$ a nonnegative lower bound for $\sigma_{n, k}$ which is independent of $n$, when $x^{n} \notin E$.

Assume that there is some $n \in \mathbb{N}^{*}$ such that $x^{n} \notin E$. This means that there exists $k_{n} \in \mathcal{N} \backslash \operatorname{esupp}(\bar{x}) \operatorname{such}$ that $x_{k_{n}}^{n} \neq 0$. Also, since $\operatorname{supp}(\bar{x}) \subset \operatorname{esupp}(\bar{x})$, we must have $\bar{x}_{k_{n}}=0$, meaning that $T_{\lambda h}(\bar{x})_{k_{n}}=-\lambda \nabla h(\bar{x})_{k_{n}}$. We deduce from (3.5), (3.6), and Lemma A.4, that

$$
T_{\lambda h}\left(x^{n-1}\right)_{k_{n}} \notin \lambda I_{k_{n}} \text { and } T_{\lambda h}(\bar{x})_{k_{n}} \in \operatorname{int} \lambda I_{k_{n}}
$$

Since Id $-\operatorname{soft}_{\lambda I_{k_{n}}}$ is the projection on $\lambda I_{k_{n}}$, we derive from (3.8) that

$$
\sigma_{n, k_{n}}=\left|\operatorname{proj}_{\lambda I_{k_{n}}}\left(T_{\lambda h}\left(x^{n-1}\right)_{k_{n}}\right)-T_{\lambda h}(\bar{x})_{k_{n}}\right|
$$

Moreover $\operatorname{proj}_{\lambda I_{k_{n}}}\left(T_{\lambda h}\left(x^{n-1}\right)_{k_{n}}\right) \in \operatorname{bd} I_{k_{n}}$, therefore by Definition 3.8 and (3.8), we obtain that

$$
\sigma_{n, k_{n}} \geq \lambda \operatorname{dist}\left(\lambda^{-1} T_{\lambda h}(\bar{x})_{k_{n}}, \operatorname{bd} I_{k_{n}}\right) \geq \lambda \rho\left(\lambda^{-1} T_{\lambda h}(\bar{x})\right)=\lambda \rho(-\nabla h(\bar{x}))=\lambda \rho_{\text {sol }} .
$$

Plugging this into (3.7), we obtain

$$
\forall n \in \mathbb{N}^{*}, x^{n} \notin E \Rightarrow\left\|x^{n}-\bar{x}\right\|^{2} \leq\left\|x^{n-1}-\bar{x}\right\|^{2}-\rho_{\text {sol }}^{2} \lambda^{2} .
$$


Next, note that the sequence $\left(x^{n}\right)_{n \in \mathbb{N}}$ is Féjer monotone with respect to the minimizers of $f$ (see e.g. [20], Thm. 2.2) - meaning that $\left(\left\|x^{n}-\bar{x}\right\|\right)_{n \in \mathbb{N}}$ is a decreasing sequence. Therefore, the inequality (3.9) cannot hold an infinite number of times. More precisely, $x_{n} \notin E$ can hold for at most $\lambda^{-2} \rho_{\text {sol }}^{-2}\left\|x^{0}-\bar{x}\right\|^{2}$ iterations.

\section{Strong CONVERGEnCE AND RATES}

\subsection{General results for thresholding gradient methods}

Strong convergence of the iterates for the thresholding gradient algorithm (introduced in Sect. 2) was first stated in ([11], Sect. 3.2) for $g=\|\cdot\|_{1}$, and then generalized to general thresholding gradient methods in ([9], Thm. 4.5). We provide a new and simple proof for this result, exploiting the "finite-dimensionality" provided by the identification result in Theorem 3.10.

Corollary 4.1 (Finite dimensionality for thresholding gradient methods). Let $\left(x^{n}\right)_{n \in \mathbb{N}}$ be generated by a thresholding gradient algorithm. Then:

(i) There exists a finite set $J \subset \mathcal{N}$ such that $x^{n} \in X_{J}$ for all $n \in \mathbb{N}^{*}$.

(ii) $x^{n}$ converges strongly to some $\bar{x} \in \operatorname{argmin} f$.

Proof. (i): Let $x \in \operatorname{argmin} f$ and let

$$
J=\operatorname{esupp}(x) \bigcup\left\{\operatorname{supp}\left(x^{n}\right) \mid n \in \mathbb{N}^{*}, x^{n} \notin X_{\operatorname{esupp}(x)}\right\},
$$

and observe that it is finite, as a finite union of finite sets (see Prop. 3.2 and Thm. 3.10).

(ii): It is well known that $\operatorname{argmin} f \neq \emptyset$ implies that $\left(x^{n}\right)_{n \in \mathbb{N}}$ converges weakly towards some $\bar{x} \in \operatorname{argmin} f$ (see e.g. [20], Thm. 2.2). In particular, $\left(x^{n}\right)_{n \in \mathbb{N}}$ is a bounded sequence in $X$. Moreover, (i) implies that $\left(x^{n}\right)_{n \in \mathbb{N}^{*}}$ belongs to $X_{J}$, which is finite-dimensional. This two facts imply that $\left(x^{n}\right)_{n \in \mathbb{N}^{*}}$ is contained in a compact set of $X$ with respect to the strong topology, and thus converges strongly.

Next we discuss the rate of convergence for the thresholding gradient methods. Beforehand, we briefly recall how the geometry of a function around its minimizers is related to the rates of convergence of the ForwardBackward algorithm.

Definition 4.2. Let $p \in\left[2,+\infty\left[\right.\right.$ and $\Omega \subset X$. We say that $\phi \in \Gamma_{0}(X)$ is $p$-conditioned on $\Omega$ if

$$
\left(\exists \gamma_{\phi, \Omega}>0\right)(\forall x \in \Omega) \quad \frac{\gamma_{\phi, \Omega}}{p} \operatorname{dist}(x, \operatorname{argmin} \phi)^{p} \leq \phi(x)-\inf \phi .
$$

A $p$-conditioned function is a function which somehow grows like $\operatorname{dist}(\cdot, \operatorname{argmin} \phi)^{p}$ on a set. For instance, strongly convex functions are 2-conditioned on $\Omega=X$, and the constant $\gamma_{\phi, X}$ is nothing but the constant of strong convexity. But the notion of $p$-conditioning is more general and also describes the geometry of functions having more than one minimizer. For instance in finite dimensions, any positive quadratic function is 2-conditioned on $\Omega=X$, in which case the constant $\gamma_{\phi, X}$ is the smallest nonzero eigenvalue of the Hessian. This notion is interesting since it allows to get precise convergence rates for some algorithms (including the Forward-Backward one) [2]:

- sublinear rates if $p>2$,

- linear rates if $p=2$.

For more examples, related notions and references, we refer the interested reader to $[5,16,20]$.

Corollary 4.1 highlights the fact that the behavior of the thresholding gradient method essentially depends on the conditioning of $f$ on finitely supported subspaces. It is then natural to introduce the following notion of finite uniform conditioning. 
Definition 4.3. Let $p \in\left[2,+\infty\left[\right.\right.$. We say that a function $\phi \in \Gamma_{0}(X)$ satisfies the finite uniform conditioning property of order $p$ if, for every finite $J \subset \mathcal{N}, \forall \bar{x} \in \operatorname{argmin} \phi, \forall(\delta, r) \in] 0,+\infty\left[^{2}, \phi\right.$ is $p$-conditioned on $X_{J} \cap$ $\mathbb{B}_{X}(\bar{x}, \delta) \cap S_{\phi}(r)$.

Remark 4.4. In this definition, we only need information about $\phi$ over supports $J$ satisfying $\operatorname{argmin} \phi \cap X_{J} \neq \emptyset$. Indeed, if $\operatorname{argmin} \phi \cap X_{J}=\emptyset$, then $\phi$ is $p$-conditioned on $X_{J} \cap \mathbb{B}_{X}(\bar{x}, \delta) \cap S_{\phi}(r)$ for any $(\delta, r)$ and for all $p \in[2,+\infty[$ according to ([20], Prop. 3.4).

In the following theorem, we illustrate how finite uniform conditioning guarantees global rates of convergence for the thresholding gradient methods: linear rates if $p=2$, and sublinear rates for $p>2$. Note that these sublinear rates are better than the $O\left(n^{-1}\right)$ rate guaranteed in the worst case.

Theorem 4.5 (Convergence rates for thresholding gradient methods). Let $\left(x^{n}\right)_{n \in \mathbb{N}}$ be generated by the ForwardBackward algorithm (FB), and let $\bar{x} \in \operatorname{argmin} f$ be its (weak) limit. Then the following hold.

(i) If $f$ satisfies the finite uniform conditioning property of order 2 , then there exist $\varepsilon \in] 0,1[$ and $C \in] 0,+\infty[$, depending on $\left(\lambda, f, x^{0}\right)$, such that

$$
(\forall n \geq 1) \quad f\left(x^{n}\right)-\inf f \leq \varepsilon^{n}\left(f\left(x^{0}\right)-\inf f\right) \quad \text { and } \quad\left\|x^{n+1}-\bar{x}\right\| \leq C \sqrt{\varepsilon}^{n} .
$$

(ii) If $f$ satisfies the finite uniform conditioning property of order $p>2$, then there exist $\left.\left(C_{1}, C_{2}\right) \in\right] 0,+\infty\left[^{2}\right.$, depending on $\left(\lambda, f, x^{0}\right)$, such that

$$
(\forall n \geq 1) \quad f\left(x^{n}\right)-\inf f \leq C_{1} n^{-\frac{p}{p-2}} \quad \text { and } \quad\left\|x_{n+1}-x_{\infty}\right\| \leq C_{2} n^{-\frac{1}{p-2}} .
$$

Proof. According to Corollary 4.1, there exists a finite set $J \subset \mathcal{N}$ such that for all $n \geq 1, x^{n} \in X_{J}$, and $x^{n}$ converges strongly to $\bar{x} \in \operatorname{argmin} f$. Also, the decreasing and Féjer properties of the Forward-Backward algorithm (see e.g. [20], Thm. 2.2) tells us that for all $n \in \mathbb{N}, x^{n} \in \mathbb{B}_{X}(\bar{x}, \delta) \cap S_{f}(r)$, by taking $\delta=\left\|x^{0}-\bar{x}\right\|$ and $r=f\left(x^{0}\right)-\inf f$. Therefore, thanks to the finite uniform conditioning assumption, we can apply ([20], Thm. 4.2) to the sequence $\left(x^{n+1}\right)_{n \in \mathbb{N}} \subset \Omega=X_{J} \cap \mathbb{B}_{X}(\bar{x}, \delta) \cap S_{f}(r)$ and conclude.

\section{2. $\ell^{1}$ regularized least squares}

Let $A: X \rightarrow Y$ be a linear operator from $X$ to a separable Hilbert space $Y$, and let $y \in Y$. In this section, we discuss the particular case when $h(x)=\frac{1}{2}\|A x-y\|_{Y}^{2}$ and $\psi \equiv 0$. The function in (P) then becomes

$$
X \ni x \mapsto f(x)=\|x\|_{1, \mathcal{I}}+\frac{1}{2}\|A x-y\|_{Y}^{2},
$$

and the Forward-Backward algorithm specializes to the ISTA. In this special case, linear convergence rates have been studied under additional assumptions on the operator $A$. A common one is injectivity of $A$ or, more generally, the so-called finite basis injectivity property (FBI) [8]. The FBI requires $A$ to be injective once restricted to $X_{J}$, for any finite $J \subset \mathcal{N}$. It is clear that the FBI property implies that $h$ is a strongly convex function once restricted to each $X_{J}$, meaning that the finite uniform conditioning of order 2 holds. So, the linear rates obtained in ([8], Thm. 1) under the FBI assumption can be directly derived from Theorem 4.5. However, as can be seen in Theorem 4.5, strong convexity is not necessary to get linear rates, and the finite uniform 2-conditioning is a sufficient condition (and it is actually necessary, see [20], Prop. 4.18). By using Li's theorem on convex piecewise polynomials ([25], Cor. 3.6), we show in Proposition 4.7 below that $f$ satisfies a finite uniform conditioning of order 2 on finitely supported subsets, without doing any assumption on the problem. First, we need a technical lemma which establishes the link between the conditioning of a function on a finitely supported space and the conditioning of its restriction to this space. 
Lemma 4.6. Let $\phi \in \Gamma_{0}(X)$, let $m \in \mathbb{N}^{*}$ and let $J=\left\{k_{1}, \ldots, k_{m}\right\} \subset \mathcal{N}$. Suppose that $\bar{x} \in \operatorname{argmin} \phi \cap X_{J}$. Let $\Xi: \mathbb{R}^{m} \rightarrow X_{J}:\left(u_{1}, \ldots, u_{m}\right) \mapsto \sum_{i=1}^{m} u_{i} e_{k_{i}}$. Assume that, for every $\left.(\delta, r) \in\right] 0,+\infty\left[{ }^{2}\right.$,

$$
\phi_{J}=\phi \circ \Xi \in \Gamma_{0}\left(\mathbb{R}^{m}\right) \text { is p-conditioned on } \mathbb{B}_{\mathbb{R}^{m}}\left(\Xi^{-1}(\bar{x}), \delta\right) \cap S_{\phi_{J}}(r) \text {. }
$$

Then $\phi$ is $p$-conditioned on $X_{J}$.

Proof. Assume without loss of generality that $k_{1}<\ldots<k_{m}$. Also, observe that $\bar{x} \in X_{J} \cap \operatorname{argmin} \phi$ implies that $\bar{u}=\Xi^{-1}(\bar{x})$ is well-defined. By definition, $\inf \phi \leq \inf \phi_{J}$, and

$$
\inf \phi=\phi(\bar{x})=\phi \circ \Xi(\bar{u})=\phi_{J}(\bar{u}) \geq \inf \phi_{J}
$$

which implies $\inf \phi=\inf \phi_{J}$. Also, we have

$$
x \in \Xi\left(\operatorname{argmin} \phi_{J}\right) \Leftrightarrow x=\Xi(u) \text { and } \phi_{J}(u)=\inf \phi_{J} \Leftrightarrow x \in X_{J} \text { and } \phi(x)=\inf \phi,
$$

meaning that $\Xi\left(\operatorname{argmin} \phi_{J}\right)=\operatorname{argmin} \phi \cap X_{J}$. Let $\left.(\delta, r) \in\right] 0,+\infty\left[^{2}\right.$, and let $\Omega=X_{J} \cap \mathbb{B}_{X}(\bar{x}, \delta) \cap S_{\phi}(r)$. Since $\phi_{J}$ is $p$-conditioned on $\mathbb{B}_{\mathbb{R}^{m}}\left(\Xi^{-1}(\bar{x}), \delta\right) \cap S_{\phi_{J}}(r)$ there exists $\left.\gamma \in\right] 0,+\infty[$ such that

$$
\left(\forall u \in \mathbb{B}_{\mathbb{R}^{m}}(\bar{u}, \delta) \cap S_{\phi_{J}}(r)\right) \quad \frac{\gamma}{p} \operatorname{dist}\left(u, \operatorname{argmin} \phi_{J}\right)^{p} \leq \phi_{J}(u)-\inf \phi_{J}
$$

Let $x=\Xi(u)$ in (4.1). Since $\|\Xi\|=1$, it is easy to see that $\|x-\bar{x}\| \leq \delta$ and $\phi(x)-\inf \phi<r$. So, we can rewrite (4.1) as:

$$
(\forall x \in \Omega) \quad \frac{\gamma}{p} \operatorname{dist}\left(\Xi^{-1} x, \operatorname{argmin} \phi_{J}\right)^{p} \leq \phi(x)-\inf \phi .
$$

It follows from $\Xi\left(\operatorname{argmin} \phi_{J}\right)=\operatorname{argmin} \phi \cap X_{J}$ that

$$
(\forall x \in \Omega) \quad \phi(x)-\inf \phi \geq \frac{\gamma}{p} \operatorname{dist}\left(x, \operatorname{argmin} \phi \cap X_{J}\right)^{p} \geq \frac{\gamma}{p} \operatorname{dist}(x, \operatorname{argmin} f)^{p} .
$$

Therefore $\phi$ is $p$-conditioned on $\Omega$.

Proposition 4.7 (Conditioning of $\ell^{1}$ regularized least squares). Let $\left(Y,\|\cdot\|_{Y}\right)$ be a separable Hilbert space, let $y \in Y$ and let $A: X \rightarrow Y$ be a bounded linear operator. In assumption $(\mathrm{H})$ suppose that for every $k \in \mathcal{N}, I_{k} \in \mathcal{I}$ is bounded. Then $X \ni x \mapsto f(x)=\|x\|_{1, \mathcal{I}}+\frac{1}{2}\|A x-y\|_{Y}^{2}$ has a finite uniform conditioning of order 2 .

Proof. Let $J \subset \mathcal{N}, J=\left\{k_{1}, \ldots, k_{m}\right\}$, with $k_{1}<\ldots<k_{m}$, and suppose that $\operatorname{argmin} f \cap X_{J} \neq \varnothing$. Define, using the same notation as in Lemma 4.6

$$
h_{J}: \mathbb{R}^{m} \rightarrow \mathbb{R}: u \mapsto \frac{1}{2}\|A \Xi u-y\|_{Y}^{2} .
$$

Define $A_{J}=A \Xi: \mathbb{R}^{m} \rightarrow Y$, and let $S_{J}=\left(A_{J}^{*} A_{J}\right)^{1 / 2}$, which verifies $R\left(S_{J}^{*}\right)=R\left(A_{J}^{*}\right)$. Thus, there exists $y_{J} \in \mathbb{R}^{m}$ such that $A_{J}^{*} y=S_{J}^{*} y_{J}$, so that we can rewrite

$$
h_{J}(u)=\frac{1}{2}\left\|A_{J} u\right\|_{\mathbb{R}^{m}}^{2}+\frac{1}{2}\|y\|_{Y}^{2}-\left\langle A_{J} u, y\right\rangle_{Y}=\frac{1}{2}\left\|S_{J} u-y_{J}\right\|_{\mathbb{R}^{m}}^{2}+\frac{1}{2}\left(\|y\|_{Y}^{2}-\left\|y_{J}\right\|_{\mathbb{R}^{m}}^{2}\right) .
$$


Set $s_{k}=S_{J} e_{k} \in Y$. Then, (4.2) yields

$$
f_{J}(u)=\sum_{i=1}^{m} \sigma_{I_{k_{i}}}\left(u_{i}\right)+\frac{1}{2} \sum_{i, j=1}^{m}\left\langle s_{k_{i}}, s_{k_{j}}\right\rangle_{Y} u_{i} u_{j}-\sum_{i=1}^{m}\left(S_{J}^{*} y_{J}\right)_{i} u_{i}+\frac{1}{2}\|y\|_{Y}^{2} .
$$

Since the intervals $I_{k}$ are bounded, their support functions are finite valued and piecewise linear, so $f_{J}$ is a piecewise polynomial of degree two in $\mathbb{R}^{m}$. We then apply ([25], Cor. 3.6) to derive that $f_{J}$ is 2-conditioned on $S_{f_{J}}(r)$, for any $\left.r \in\right] 0,+\infty[$. We conclude by using Lemma 4.6 .

Combining Theorem 4.5 and Proposition 4.7, we can now state our main result concerning the linear rates of ISTA.

Theorem 4.8 (Linear convergence for the iterative soft thresholding). Under the assumptions of Proposition 4.7 , let $\left(x^{n}\right)_{n \in \mathbb{N}}$ be the sequence generated by the Forward-Backward algorithm applied to $f$. Then $\left(x^{n}\right)_{n \in \mathbb{N}}$ converges strongly to some $\bar{x} \in \operatorname{argmin} f$, and there exists two constants $\varepsilon \in] 0,1[$ and $C \in] 0,+\infty[$, depending on $\left(\lambda, L, x^{0}, \mathcal{I}, A, y\right)$, such that

$$
(\forall n \geq 1) \quad f\left(x^{n}\right)-\inf f \leq \varepsilon^{n}\left(f\left(x^{0}\right)-\inf f\right) \quad \text { and } \quad\left\|x^{n+1}-\bar{x}\right\| \leq C \sqrt{\varepsilon}^{n} .
$$

Remark 4.9 (On the linear rates). The convergence rate for the iterative soft-thresholding has been a subject of interest since years, and has been obtained only under additional assumptions on $A$ [8]. Theorem 4.8 closes the question about the linear rates, by proving that they always hold. However, there are still several open problems, related to the estimation of the constant appearing in these linear rates. This is related to the estimation of the constant $\gamma_{f, \Omega}$ in Definition 4.2, when $\Omega=S_{f}(r) \cap X_{J}$ for some finite $J \subset \mathcal{N}$. Up to now, the only available result is based on Hoffman's lemma, which does not allow for explicit lower bounds on $\gamma_{f, \Omega}[6,29]$. Having a tight lower bound for $\gamma_{f, \Omega}$, depending on $A$ restricted to $X_{J}$, would be of interest to go in this direction.

\section{3. $\ell^{1}+\ell^{p}$ regularized least squares}

We are now interested in $\ell^{1}+\ell^{p}$-regularizers, i.e. when

$$
g(x)=\|x\|_{1, \mathcal{I}}+\frac{1}{p}\|x\|^{p}, \text { with }\|x\|^{p}=\sum_{k \in \mathbb{N}}\left|x_{k}\right|^{p}, \quad p>1 .
$$

The case $p=2$ is also known as elastic net regularization and has been proposed in [35]. The elastic-net penalty has been studied by the statistical machine learning community as an alternative to the $\ell^{1}$ regularization in variable selection problems where there are highly correlated features and all the relevant ones have to be identified [13]. See also [10] for the case $p<2$. Note that the proximal operator of such $g$ can be computed explicitly when $p \in\{4 / 3,3 / 2,2,3,4\}$ (see [9]).

Proposition 4.10 (Geometry of $\left(\ell^{1}+\ell^{p}\right)$ regularized least squares). Let $\left.p \in\right] 1,+\infty\left[\right.$, let $\left(Y,\|\cdot\|_{Y}\right)$ be a separable Hilbert space, let $y \in Y$ and let $A: X \rightarrow Y$ be a bounded linear operator. In assumption $(\mathrm{H})$ suppose that for every $k \in \mathcal{N}, I_{k} \in \mathcal{I}$ is bounded. Then $f: X \rightarrow \mathbb{R}: x \mapsto\|x\|_{1, \mathcal{I}}+\frac{1}{p}\|x\|_{p}^{p}+\frac{1}{2}\|A x-y\|_{Y}^{2}$ has a finite uniform conditioning of order $\max \{2, p\}$.

Proof. Let $J \subset \mathcal{N}, m=|J|$ and $p^{\prime}=\max \{p, 2\}$. We define, by using the same notation as in Lemma 4.6,

$$
g_{J}(u)=\sum_{i=1}^{m} \sigma_{I_{k_{i}}}\left(u_{i}\right)+\frac{1}{p}\left|u_{i}\right|^{p} \text { and } h_{J}(u)=\frac{1}{2}\|A \Xi u-y\|_{Y}^{2} .
$$


We are going to prove that $f_{J}=g_{J}+h_{J}$ is $p^{\prime}$-conditioned on $\mathbb{B}_{\mathbb{R}^{m}}(\bar{u}, \delta)$, for any $\delta>0$. To do so, we will apply to $f_{J}$ the sum rule in Theorem A.7, which requires two hypotheses. We must verify that the functions $g_{J}$ and $h_{J}$ are conditioned up to linear perturbations (see Eq. (A.2)), and that the qualification condition in (A.3) holds, namely (since $\mathbb{R}^{m}$ is finite-dimensional the strong relative interior coincides with the relative interior):

$$
0 \in \operatorname{ri}\left(\partial g_{J}^{*}\left(-\nabla h_{J}(\bar{u})\right)-\partial h_{J}^{*}\left(\nabla h_{J}(\bar{u})\right)\right)
$$

According to (4.2), $\partial h_{J}^{*}\left(\nabla h_{J}(\bar{u})\right)=\bar{u}+\operatorname{ker} S_{J}$. Also, according to ([4], Prop. 13.30 and Ex. 13.27(iii)), we have, for every $v \in \mathbb{R}^{m}, g_{J}^{*}(v)=\sum_{i=1}^{m} \frac{1}{q} \operatorname{dist}\left(v_{i}, I_{k_{i}}\right)^{q}$, with $q=p /(p-1)$. Since $t \mapsto|t|^{q}$ is continuously differentiable on $\mathbb{R}$ ([4], Ex. 17.33 and Prop. 17.31(ii)) imply that $g_{J}^{*}$ is Gâteaux differentiable on $\mathbb{R}^{m}$. This, together with the fact that ri $\operatorname{ker} S_{J}=\operatorname{ker} S_{J}$, means that (4.3) is equivalent to

$$
0 \in \operatorname{ri}\left(\nabla g_{J}^{*}\left(-\nabla h_{J}(\bar{u})\right)-\bar{u}+\operatorname{ker} S_{J}\right)=\nabla g_{J}^{*}\left(-\nabla h_{J}(\bar{u})\right)-\bar{u}+\operatorname{ker} S_{J}
$$

The latter inclusion holds true, since $\bar{u} \in \operatorname{argmin} f_{J}$ is equivalent to $\bar{u} \in \nabla g_{J}^{*}\left(-\nabla h_{J}(\bar{u})\right)$. Thus, it only remains to prove that $\bar{h}_{J}=h_{J}-\langle\ell, \cdot\rangle$ and $\bar{g}_{J}=g_{J}-\langle\ell, \cdot\rangle$ are respectively 2 and $p^{\prime}$-conditioned on $\mathbb{B}_{\mathbb{R}^{m}}(\bar{u}, \delta)$, for $\ell$ being respectively in $R\left(\nabla h_{J}\right)$ and $R\left(\partial g_{J}\right)$.

Let us start with $\bar{h}_{J}$. According to (4.2), $\bar{h}_{J}$ is a positive quadratic function bounded from below, so it is 2-conditioned on $\mathbb{R}^{m}$, with $\gamma_{\bar{h}_{J}, \mathbb{R}^{m}}$ being the smallest nonzero eigenvalue of $S_{J}$. Next, $\ell \in R\left(\partial g_{J}\right)$ implies that there exists $v \in X$ such that $\ell \in \partial \bar{g}_{J}(v)$. Then, $0 \in \partial \bar{g}_{J}((v))$, and this implies that $v$ is a minimizer of $\bar{g}_{J}$. It is also unique since $g_{j}$ is strictly convex. If $v \notin \mathbb{B}_{\mathbb{R}^{m}}(\bar{u}, \delta)$, then $\bar{g}_{J}$ is automatically $p^{\prime}$-conditioned on $\mathbb{B}_{\mathbb{R}^{m}}(\bar{u}, \delta)$, see for instance ([20], Prop. 3.3). Assume then that $v \in \mathbb{B}_{\mathbb{R}^{m}}(\bar{u}, \delta)$, and use ([10], Prop. A.9) to obtain the existence of $\gamma \in] 0,+\infty[$ such that

$$
\left(\forall u \in \mathbb{B}_{\mathbb{R}^{m}}(\bar{u}, \delta)\right)(\forall i \in\{1, \ldots, m\}) \quad \frac{\gamma}{p^{\prime}}\left|u_{i}-v_{i}\right|^{p^{\prime}} \leq \frac{1}{p}\left|u_{i}\right|^{p}-\frac{1}{p}\left|v_{i}\right|^{p}-\left(u_{i}-v_{i}\right) \operatorname{sgn}\left(v_{i}\right)\left|v_{i}\right|^{p-1}
$$

Summing the above inequality over $i$, and using the fact that $\|\cdot\|_{2}^{p^{\prime}} \leq \max \left\{1, m^{(p-2) / 2}\right\}\|\cdot\|_{p^{\prime}}^{p^{\prime}}$, we derive by taking $\gamma^{\prime}=\gamma \max \left\{1, m^{(p-2) / 2}\right\}^{-1}$ that for all $u \in \mathbb{B}_{\mathbb{R}^{m}}(\bar{u}, \delta)$ :

$$
\frac{\gamma^{\prime}}{p^{\prime}} \operatorname{dist}\left(u, \operatorname{argmin} \bar{g}_{J}\right)^{p^{\prime}} \leq \sum_{i=1}^{m} \frac{1}{p}\left|u_{i}\right|^{p}-\frac{1}{p}\left|v_{i}\right|^{p}-\left(u_{i}-v_{i}\right) \operatorname{sgn}\left(v_{i}\right)\left|v_{i}\right|^{p-1} .
$$

Introduce the following constant: $\omega_{i}=\sup I_{k_{i}}$ if $\ell_{i}>\sup I_{k_{i}}, \omega_{i}=\left|\ell_{i}\right|$ if $\ell_{i} \in I_{k_{i}}$, and $w_{i}=-\inf I_{k_{i}}$ if $\ell_{i}<\inf I_{k_{i}}$. By making use of the first order condition at $v=\operatorname{argmin} \bar{g}_{J}$, it can be verified that

$$
\left(\forall_{i} \in\{1, \ldots, m\}\right) \quad\left|v_{i}\right|^{p-1}=\left|\ell_{i}\right|-\omega_{i}, \operatorname{sgn}\left(v_{i}\right)=\operatorname{sgn}\left(\ell_{i}\right) \text { and } \sigma_{I_{k_{i}}}\left(v_{i}\right)=\omega_{i}\left|v_{i}\right|
$$

So we can deduce that

$$
\begin{aligned}
& \frac{1}{p}\left|u_{i}\right|^{p}-\frac{1}{p}\left|v_{i}\right|^{p}-\left(u_{i}-v_{i}\right) \operatorname{sgn}\left(v_{i}\right)\left|v_{i}\right|^{p-1} \\
= & \frac{1}{p}\left|u_{i}\right|^{p}-\frac{1}{p}\left|v_{i}\right|^{p}-\left(u_{i}-v_{i}\right)\left(\ell_{i}-\operatorname{sgn}\left(v_{i}\right) \omega_{i}\right) \\
= & \frac{1}{p}\left|u_{i}\right|^{p}-\frac{1}{p}\left|v_{i}\right|^{p}-u_{i} \ell_{i}+v_{i} \ell_{i}-\sigma_{I_{k_{i}}}\left(v_{i}\right)+u_{i} \operatorname{sgn}\left(\ell_{i}\right) \omega_{i} .
\end{aligned}
$$


This, combined with (4.5), leads to

$$
\frac{\gamma^{\prime}}{p^{\prime}} \operatorname{dist}\left(u, \operatorname{argmin} \bar{g}_{J}\right)^{p^{\prime}} \leq \bar{g}_{J}(u)-\inf \bar{g}_{J}+\sum_{i=1}^{m}-\sigma_{I_{k_{i}}}\left(u_{i}\right)+u_{i} \operatorname{sgn}\left(\ell_{i}\right) \omega_{i} \leq \bar{g}_{J}(u)-\inf \bar{g}_{J},
$$

where the last inequality comes from the fact that $\operatorname{sgn}\left(\ell_{i}\right) \omega_{i} \in I_{k_{i}}$. So, we proved that $\bar{g}_{J}$ is $p^{\prime}$-conditioned on $\mathbb{B}_{\mathbb{R}^{m}}(\bar{u}, \delta)$. Theorem A.7 then yields that $f_{J}$ is $p^{\prime}$-conditioned on $\mathbb{B}_{\mathbb{R}^{m}}(\bar{u}, \delta)$. We conclude the proof applying Lemma 4.6.

Combining Theorem 4.5 and Proposition 4.10 be obtain rates for the corresponding thresholding gradient method.

Theorem 4.11. Under the assumptions of Proposition 4.10, let $\left(x^{n}\right)_{n \in \mathbb{N}}$ be the sequence generated by the Forward-Backward algorithm applied to $f$. Then $\left(x^{n}\right)_{n \in \mathbb{N}}$ converges strongly to some $\bar{x} \in \operatorname{argmin} f$. If $\left.p \in\right] 1,2[$, there exists two constants $\varepsilon \in] 0,1[$ and $C \in] 0,+\infty\left[\right.$, depending on $\left(\lambda, L, x^{0}, \mathcal{I}, A, y, p\right)$, such that

$$
(\forall n \geq 1) \quad f\left(x^{n}\right)-\inf f \leq \varepsilon^{n}\left(f\left(x^{0}\right)-\inf f\right) \quad \text { and } \quad\left\|x^{n+1}-\bar{x}\right\| \leq C \sqrt{\varepsilon}^{n} .
$$

If $p \in] 2,+\infty\left[\right.$, there exists two constants $\left.\left(C_{1}, C_{2}\right) \in\right] 0,+\infty\left[{ }^{2}\right.$, depending on $\left(\lambda, L, x^{0}, \mathcal{I}, A, y, p\right)$, such that

$$
(\forall n \geq 1) \quad f\left(x^{n}\right)-\inf f \leq C_{1} n^{-\frac{p}{p-2}} \quad \text { and } \quad\left\|x_{n+1}-x_{\infty}\right\| \leq C_{2} n^{-\frac{1}{p-2}} .
$$

\section{CONCLUSION AND PERSPECTIVES}

In this paper, we study and highlight the importance of the notion of extended support for minimization problems with sparsity inducing separable penalties. An identification result, together with uniform conditioning on finite-dimensional sets, allows us to generalize and revisit classic convergence results for thresholding gradient methods, from a novel and different perspective, while further providing new convergence rates. An interesting direction for future research would be to go beyond separable penalties, in particular extending our results to regularizers promoting structured sparsity [28], such as group lasso. A reasonable approach would be to extend the primal-dual arguments in [18] to the infinite-dimensional setting.

A more challenging research direction would be to study the strong convergence, as well as finite identification, for algorithms used to solve gridless problems [17]. Indeed, our analysis relies strongly on the fact that the variables (signals) we consider are supported on a grid (indexed by $\mathcal{N} \subset \mathbb{N}$ ), which allows to exploit finitedimensional arguments. For such an extension, we would need to work on a Banach space of measures, in which our arguments concerning finite identification of the (extended) support would not apply. Indeed, the support of the solution $\bar{x}$ can in general be not finite. Nevertheless, it is possible under certain modeling assumptions to ensure that $\bar{x}$ has a finite support and extended support ([17], Thm. 2), or to have some control on the size of its support ([17], Lem. 1). It would be an interesting venue for future research to extend part of our arguments in this context.

\section{Appendix A. Annex}

\section{A.1 Closure, interior, boundary}

Proposition A.1. Let $C \subset X$ be a closed convex set. Then:

(i) $\partial \sigma_{C}(0)=C$.

(ii) For all $d \in X \backslash\{0\}, \quad \partial \sigma_{C}(d) \subset \operatorname{bd} C$.

Assume moreover that $\operatorname{int} C \neq \emptyset$.

(iii) $\operatorname{int} C=\operatorname{qri} C$. 
Proof. (i): see ([4], Ex. 16.34).

(ii): see ([4], Prop. 7.3 and Thm. 7.4).

(iii): see ([4], Fact 6.14).

Proposition A.2. Let $\mathcal{I}=\left(I_{k}\right)_{k \in \mathcal{N}}$ be a collection of closed proper intervals of $\mathbb{R}$, and suppose that $[-\omega, \omega] \subset I_{k}$ for all $k \in \mathcal{N}$. For every $x \in X$, let

$$
\rho(x)=\inf _{x_{k} \in \operatorname{int} I_{k}} \operatorname{dist}\left(x_{k}, \operatorname{bd} I_{k}\right) .
$$

Then the following hold

(i) For every $x \in X$ with $\operatorname{supp}(x) \neq \mathcal{N}, \quad \rho(x) \in] 0,+\infty[$;

(ii) int $\left(\bigoplus_{k \in \mathcal{N}} I_{k}\right)=\bigoplus_{k \in \mathcal{N}} \operatorname{int} I_{k}$.

Proof. (i) Let $x \in X$. If $\mathcal{N}$ is finite the statement follows immediately. If $\mathcal{N}$ is infinite, since $\left|x_{k}\right|$ tends to 0 when $k \rightarrow+\infty$, there exists $K \in \mathcal{N}$ such that for all $k \geq K,\left|u_{k}\right| \leq \omega / 2$. Now, consider the following subsets of $\mathcal{N}$

$$
J=\left\{k \in \mathcal{N} \mid u_{k} \in \operatorname{int} I_{k}\right\}, J_{F}=J \cap\{0, \ldots, K-1\}, J_{\infty}=J \backslash J_{F},
$$

which are defined in such a way that $\rho(x)=\inf _{k \in J} \operatorname{dist}\left(u_{k}\right.$, bd $\left.I_{k}\right)$ and $J=J_{F} \sqcup J_{\infty}$. Observe that $\rho(x) \leq$ $\operatorname{dist}\left(x_{K}\right.$, bd $\left.I_{K}\right)<+\infty$ since bd $I_{K} \neq \varnothing$, so we only need to show that $\rho(x)>0$. Since $J_{F}$ is finite and $x_{k} \in \operatorname{int} I_{k}$ for all $k \in J_{F}$, we have $\operatorname{dist}\left(x_{k}, \operatorname{bd} I_{k}\right)>0$ for all $k \in J_{F}$. So, we deduce that $\inf _{k \in J_{F}} \operatorname{dist}\left(x_{k}, \operatorname{bd} I_{k}\right)>0$. On the other hand, for any $k \in J_{\infty}$, we have $\left|u_{k}\right| \leq \omega / 2$, while $[-\omega, \omega] \subset I_{k}$, therefore $\operatorname{dist}\left(x_{k}, \operatorname{bd} I_{k}\right) \geq \omega / 2$, and $\rho(x)=\inf _{k \in J} \operatorname{dist}\left(x_{k}, \operatorname{bd} I_{k}\right)>0$.

(ii) Let $x \in \operatorname{int} \bigoplus_{k \in \mathcal{N}} I_{k}$. We are going to show that $x_{k} \in \operatorname{int} I_{k}$ for all $k \in \mathcal{N}$. By assumption, there exists $\delta \in] 0,+\infty\left[\right.$ such that $\mathbb{B}_{X}(x, \delta) \subset \bigoplus_{k \in \mathcal{N}} I_{k}$. Let $k \in \mathcal{N}$, and let us show that $\left[x_{k}-\delta, x_{k}+\delta\right] \subset I_{k}$. Let $y_{k} \in\left[x_{k}-\delta, x_{k}+\delta\right]$, and define $\bar{x} \in X$ such that $\bar{x}_{k}=y_{k}$ and $\bar{x}_{i}=x_{i}$ for every $i \neq k$. Then we derive that $\|x-\bar{x}\|=\left|x_{k}-y_{k}\right|=\delta$, whence $\bar{x} \in \mathbb{B}_{X}(x, \delta) \subset \bigoplus_{k \in \mathcal{N}} I_{k}$. This implies that $y_{k} \in I_{k}$, which proves that $x_{k} \in \operatorname{int} I_{k}$. Now, we let $x \in \bigoplus_{k \in \mathcal{N}} \operatorname{int} I_{k}$, and we show that $x \in \operatorname{int}\left(\bigoplus_{k \in \mathcal{N}} I_{k}\right)$. By (i), $\rho(x)>0$. Let $\eta \in] 0, \rho(x)\left[\right.$. Since for every $k \in \mathcal{N}, x_{k} \in \operatorname{int} I_{k}$ by assumption, then $\operatorname{dist}\left(x_{k}\right.$, bd $\left.I_{k}\right) \geq \rho(x)$, and we derive that $\left[x_{k}-\eta, x_{k}+\eta\right] \subset \operatorname{int} I_{k}$. Next, note that $\mathbb{B}_{X}(x, \eta) \subset \bigoplus_{k \in \mathcal{N}}\left[x_{k}-\eta, x_{k}+\eta\right]$ and the statement follows.

Proposition A.3 (Quasi relative interior of infinite products). Let $\mathcal{I}=\left(I_{k}\right)_{k \in \mathbb{N}}$ be a collection of closed intervals of $\mathbb{R}$. Let $J \subset \mathcal{N}$ be a finite set, and suppose that $[-\omega, \omega] \subset I_{k}$ for all $k \in \mathcal{N} \backslash J$. Then

$$
\operatorname{qri} \bigoplus_{k \in \mathcal{N}} I_{k}=\bigoplus_{k \in \mathcal{N}} \operatorname{ri} I_{k}
$$

Proof. Assume $\mathcal{N}$ is infinite and set $J_{\infty}=\mathcal{N} \backslash J$. We can then write

$$
\begin{aligned}
& \text { qri } \bigoplus_{k \in \mathcal{N}} I_{k}=\quad \operatorname{qri}\left(\left(\bigoplus_{k \in J_{\infty}} I_{k}\right) \oplus\left(\bigoplus_{k \in J} I_{k}\right)\right) \quad \text { because } J \text { is finite, } \\
& =\quad \operatorname{qri}\left(\bigoplus_{k \in J_{\infty}} I_{k}\right) \oplus\left(\bigoplus_{k \in J} \operatorname{qri} I_{k}\right) \quad \text { by ([7], Prop. 2.5), } \\
& =\quad\left(\bigoplus_{k \in J_{\infty}} \operatorname{int} I_{k}\right) \oplus\left(\bigoplus_{k \in J} \text { qri } I_{k}\right) \quad \text { by Proposition A.2, } \\
& =\quad \bigoplus_{k \in \mathcal{N}} \operatorname{ri} I_{k} \quad \text { by Proposition A.1(iii). }
\end{aligned}
$$




\section{A.2 Functions}

Lemma A.4. Let $\psi \in \Gamma_{0}(X)$ be differentiable at $0 \in \operatorname{argmin} \psi$ and let $x \in X$. Then

$$
x=0 \Leftrightarrow \operatorname{prox}_{\psi}(x)=0 .
$$

Proof. $\operatorname{prox}_{\psi}(x)=0 \Leftrightarrow(I d+\partial \psi)^{-1}(x)=0 \Leftrightarrow x \in 0+\partial \psi(0) \Leftrightarrow x=\nabla \psi(0) \Leftrightarrow x=0$.

Proposition A.5. Let $g_{k} \in \Gamma_{0}(\mathbb{R})$ with inf $g_{k}=g_{k}(0)=0$ for all $k \in \mathcal{N}$. Define $g: X \rightarrow \mathbb{R} \cup\{+\infty\}: x \mapsto$ $\sum_{k \in \mathcal{N}} g_{k}\left(x_{k}\right)$. Then:

(i) $g \in \Gamma_{0}(X)$.

(ii) $\operatorname{dom} \partial g=\left\{x \in X \mid \bigoplus_{k \in \mathcal{N}} \partial g_{k}\left(x_{k}\right) \neq \emptyset\right\}$.

(iii) For all $x \in \operatorname{dom} \partial g, \partial g(x)=\bigoplus_{k \in \mathcal{N}} \partial g_{k}\left(x_{k}\right)$.

(iv) For all $x \in X, \operatorname{prox}_{g}(x)=\sum_{k \in \mathcal{N}} \operatorname{prox}_{g_{k}}\left(x_{k}\right) e_{k}$.

Proof. (i): $g$ is convex by definition. It is proper because $g(0)=0$ and $g \geq 0$. Fatou's lemma implies that $g$ is lower semicontinuous.

(ii)-(iii): follow directly from the fact that

$$
\begin{aligned}
& \left(\forall\left(x^{*}, x\right) \in X^{2}\right) \quad x^{*} \in \partial g(x) \Leftrightarrow(\forall y \in X) \quad g(y)-g(x)-\left\langle x^{*}, y-x\right\rangle \geq 0 \\
& \Leftrightarrow(\forall y \in X) \quad \sum_{k \in \mathbb{N}} g_{k}\left(y_{k}\right)-g_{k}\left(x_{k}\right)-\left\langle x_{k}^{*}, y_{k}-x_{k}\right\rangle \geq 0 \\
& \Leftrightarrow(\forall k \in \mathcal{N}) \quad x_{k}^{*} \in \partial g_{k}\left(x_{k}\right),
\end{aligned}
$$

where the last equivalence holds by taking for all $k \in \mathcal{N} \quad y_{i}=x_{i}$ if $i \neq k$.

(iv): let $(x, p) \in X^{2}$. It follows from (iii) that

$$
\begin{aligned}
p=\operatorname{prox}_{g}(x) & \Longleftrightarrow p-x \in \partial g(p) \\
& \Longrightarrow(\forall k \in \mathcal{N}) \quad p_{k}-x_{k} \in \partial g_{k}\left(p_{k}\right) \\
& \Longleftrightarrow(\forall k \in \mathcal{N}) \quad p_{k}=\operatorname{prox}_{g_{k}}\left(x_{k}\right)
\end{aligned}
$$

Proposition A.6. Let $\mathcal{I}=\left(I_{k}\right)_{k \in \mathbb{N}}$ is a family of proper closed interval of $\mathbb{R}$. Let, for every $x \in X, g(x)=$ $\sum_{k \in \mathcal{N}} \sigma_{I_{k}}\left(x_{k}\right)$. Then the following hold.

(i) $g$ is coercive if and only if $0 \in \operatorname{int} I_{k}$ for all $k \in \mathcal{N}$.

Assume moreover that there exists $\omega>0$ such that $[-\omega, \omega] \subset I_{k}$ for all $k \in \mathcal{N}$. Then

(ii) $g \in \Gamma_{0}(X)$ is coercive and $g$ is the support function of $\mathbb{B}_{\infty, \mathcal{I}}=\bigoplus_{k \in \mathcal{N}} I_{k}$,

(iii) $\operatorname{dom} \partial g=c_{00}$ and $\operatorname{dom} \partial g^{*}=\mathbb{B}_{\infty, \mathcal{I}}$,

(iv) for every $x \in X$, and for every $\lambda>0, \operatorname{prox}_{\lambda g}(x)=\left(x_{k}-\lambda \operatorname{proj}_{I_{k}}\left(\lambda^{-1} x_{k}\right)\right)_{k \in \mathcal{N}}$.

Proof. (i): observe that

$$
\begin{aligned}
g \text { is coercive } & \left.\Leftrightarrow(\forall k \in \mathcal{N}) \quad \sigma_{I_{k}} \text { is coercive } \quad \text { (take } x_{k}=0 \text { except for one index } k\right) \\
& \Leftrightarrow(\forall k \in \mathcal{N}) \quad 0 \in \operatorname{int} \operatorname{dom} \sigma_{I_{k}}^{*} \quad \text { by }([4], \text { Prop. 14.16) } \\
& \Leftrightarrow(\forall k \in \mathcal{N}) \quad 0 \in \operatorname{int} I_{k} \quad \text { since } \sigma_{I_{k}}^{*}=\delta_{I_{k}} .
\end{aligned}
$$


(ii): assume that $\mathcal{N}$ is infinite. Item (i) implies that $g \in \Gamma_{0}(X)$ and is coercive. To prove that $g$ is the support function of $\mathbb{B}_{\infty, \mathcal{I}}$, we will show that $g^{*}$ is its indicator function. Let $x^{*} \in X$. Then

$$
\begin{aligned}
g^{*}\left(x^{*}\right) & =\sup _{x \in X}\left\langle x^{*}, x\right\rangle-g(x)=\sup _{x \in X} \sum_{k \in \mathcal{N}}\left\langle x_{k}^{*}, x_{k}\right\rangle-\sigma_{I_{k}}\left(x_{k}\right) \\
& \leq \sum_{k \in \mathcal{N}} \sup _{x_{k} \in \mathbb{R}}\left\langle x_{k}^{*}, x_{k}\right\rangle-\sigma_{I_{k}}\left(x_{k}\right)=\sum_{k \in \mathcal{N}} \sigma_{I_{k}}^{*}\left(x_{k}^{*}\right)=\delta_{\mathbb{B}_{\infty, \mathcal{I}}}\left(x^{*}\right) .
\end{aligned}
$$

To prove the converse inequality, since $x^{*} \in X$, there exists some $K \in \mathcal{N}$ such that for all $k \geq K,\left\|x_{k}^{*}\right\|<\omega$, meaning that $x_{k}^{*} \in I_{k}$, and therefore $\delta_{I_{k}}\left(x_{k}^{*}\right)=0$. Let $J_{K}=\{0, \ldots, K-1\}$. Since we deal with a finite sum,

$$
\delta_{\mathbb{B}_{\infty, \mathcal{I}}}\left(x^{*}\right)=\sum_{k \in J_{K}} \delta_{I_{k}}\left(x_{k}^{*}\right)=\sum_{k \in J_{K}} \sup _{x_{k} \in \mathbb{R}}\left\langle x_{k}^{*}, x_{k}\right\rangle-\sigma_{I_{k}}\left(x_{k}\right)=\sup _{x \in X_{J_{K}}} \sum_{k \in J_{K}}\left\langle x_{k}^{*}, x_{k}\right\rangle-\sigma_{I_{k}}\left(x_{k}\right) .
$$

Moreover, setting $J_{\infty}=\mathcal{N} \backslash J_{K}$ :

$$
\sup _{x \in X_{J_{\infty}}} \sum_{k \in J_{\infty}}\left\langle x_{k}^{*}, x_{k}\right\rangle-\sigma_{I_{k}}\left(x_{k}\right) \geq 0
$$

and this yields

$$
\delta_{\mathbb{B}_{\infty, \mathcal{I}}}\left(x^{*}\right) \leq \sup _{x \in X_{J_{K}}} \sum_{k \in J_{K}}\left\langle x_{k}^{*}, x_{k}\right\rangle-\sigma_{I_{k}}\left(x_{k}\right)+\sup _{x \in X_{J_{\infty}}} \sum_{k \in J_{\infty}}\left\langle x_{k}^{*}, x_{k}\right\rangle-\sigma_{I_{k}}\left(x_{k}\right)=g^{*}\left(x^{*}\right) .
$$

(iii): assume that $\mathcal{N}$ is infinite. The equality dom $\partial g^{*}=\mathbb{B}_{\infty, \mathcal{I}}$ follows from (ii). It remains to show that dom $\partial g=$ $c_{00}$. Let $x \in \operatorname{dom} \partial g$. By Proposition A.5(ii) there exists $x^{*} \in \bigoplus_{k \in \mathcal{N}} \partial \sigma_{I_{k}}\left(x_{k}\right)$. For all $k \in \mathcal{N}$, Proposition A.1(i)(ii) yields that $\partial \sigma_{I_{k}}\left(x_{k}\right)=I_{k}$ if $x_{k}=0$, and $\partial \sigma_{I_{k}}\left(x_{k}\right) \subset$ bd $I_{k}$ if $x_{k} \neq 0$. Assume by contradiction that $x \notin c_{00}$, i.e. there exists $k_{n} \rightarrow+\infty$ such that $x_{k_{n}} \neq 0$ for all $n \in \mathbb{N}$. Then, it follows that $x_{k_{n}}^{*} \in$ bd $I_{k_{n}}$ for all $n \in \mathbb{N}$, and therefore $\left\|x_{k_{n}}^{*}\right\| \geq \omega$, which contradicts the fact that $x^{*} \in X$. Now, let $x \in c_{00}$ and let $K \in \mathcal{N}$ be such that $x_{k}=0$ for all $k \geq K$ and let $\mathcal{N}_{K}=\mathcal{N} \cap\{0, \ldots, K\}$. By Proposition A.1(i) $\partial \sigma_{I_{k}}\left(x_{k}\right)=I_{k} \ni 0$ for all $k \geq K$, therefore

$$
\emptyset \neq \bigoplus_{k \in \mathcal{N}_{K}} \partial \sigma_{I_{k}}\left(x_{k}\right) \subset \partial g(x)
$$

(iv): is a direct consequence of Proposition A.5(iv) and Moreau's identity ([4], Thm. 14.3.ii).

We recall a sum rule for conditioning, obtained in ([20], Thm. 3.1).

Theorem A.7. Let $f=g+h$, where $g \in \Gamma_{0}(X)$ and $h \in \Gamma_{0}(X)$ is of class $C^{1}$. Let $\Omega \subset X$. Assume that there exists $\bar{x} \in \operatorname{argmin} f$ such that, for $\bar{v}=-\nabla h(\bar{x})$ and $\left(p_{1}, p_{2}\right) \in\left[1,+\infty\left[^{2}\right.\right.$,

$$
g-\langle\bar{v}, \cdot\rangle \text { is } p_{1} \text {-conditioned on } \Omega \text { and } h+\langle\bar{v}, \cdot\rangle \text { is } p_{2} \text {-conditioned on } \Omega .
$$

Suppose that

$$
0 \in \operatorname{sri}\left(\partial g^{*}(\bar{v})-\partial h^{*}(-\bar{v})\right)
$$

and let $p=\max \left\{p_{1}, p_{2}\right\}$. Then, for any $\left.\delta \in\right] 0,+\infty\left[, f\right.$ is $p$-conditioned on $\Omega \cap \mathbb{B}_{X}(0, \delta)$. 


\section{A.3 Auxiliary results}

Lemma A.8. Let $\left\{x^{1}, \ldots, x^{N}\right\} \subset X$ be a finite family. Then there exists $\bar{x} \in \operatorname{co}\left\{x^{1}, \ldots, x^{N}\right\}$ such that $\operatorname{supp}(\bar{x})=$ $\cup\left\{\operatorname{supp}\left(x^{i}\right) \mid i \in\{1, \ldots, N\}\right\}$.

Proof. We proceed by induction. If $N=1$ this is trivially true. Let us turn on the $N=2$ case, by considering $\left\{x^{1}, x^{2}\right\}$ in $X$. If $\operatorname{supp}\left(x^{1}\right)=\operatorname{supp}\left(x^{2}\right)$, then it is enough to take $\bar{x}=x^{1}$ or $\bar{x}=x^{2}$. Assume that $\operatorname{supp}\left(x^{1}\right) \neq$ $\operatorname{supp}\left(x^{2}\right)$. Define

$$
\Lambda=\left\{\frac{\left|x_{k}^{2}\right|}{\left|x_{k}^{2}-x_{k}^{1}\right|} \mid k \in \mathcal{N}, x_{k}^{1} x_{k}^{2}<0\right\}
$$

$\Lambda$ is well defined because $x_{k}^{1} x_{k}^{2}<0$ implies that $x_{k}^{2}-x_{k}^{1} \neq 0$. Moreover, $\left.\Lambda \subset\right] 0,1[$, and is at most countable. Let $\lambda \in] 0,1\left[\backslash \Lambda\right.$, and define $\bar{x}=\lambda x^{1}+(1-\lambda) x^{2}$. By definition we have $\bar{x} \in \operatorname{co}\left\{x^{1}, x^{2}\right\}$, so it remains to check that $\operatorname{supp}(\bar{x})=\operatorname{supp}\left(x^{1}\right) \cup \operatorname{supp}\left(x^{2}\right)$. To prove this, first assume that $k \in \operatorname{supp}(\bar{x})$. If $k \in \operatorname{supp}\left(x^{1}\right)$ it is trivial, so assume that $k \notin \operatorname{supp}\left(x^{1}\right)$. In that case $\bar{x}_{k}=\lambda \cdot 0+(1-\lambda) x_{k}^{2}$, where $\lambda \neq 1$ and $\bar{x}_{k} \neq 0$, from which we deduce that $k \in \operatorname{supp}\left(x^{2}\right)$. This shows that $\operatorname{supp}(\bar{x}) \subset \operatorname{supp}\left(x^{1}\right) \cup \operatorname{supp}\left(x^{2}\right)$. Now, take $k \in \operatorname{supp}\left(x^{1}\right) \cup \operatorname{supp}\left(x^{2}\right)$, and assume by contradiction that $\bar{x}_{k}=0$. Then

$$
x_{k}^{1} \neq 0, x_{k}^{2} \neq 0, x_{k}^{1}=\left(1-\lambda^{-1}\right) x_{k}^{2}, \text { and } \lambda=\frac{\left|x_{k}^{2}\right|}{\left|x_{k}^{2}-x_{k}^{1}\right|},
$$

which contradicts the fact that $\lambda \notin \Lambda$. Therefore $\operatorname{supp}\left(x^{1}\right) \cup \operatorname{supp}\left(x^{2}\right) \subset \operatorname{supp}(\bar{x})$. Assume now that the statement holds for $N \geq 2$, and let us prove it for $N+1$. Let $\left\{x^{1}, \ldots, x^{N}, x^{N+1}\right\} \subset X$ be a finite family. By inductive hypotheses we can find some $\bar{x}^{1} \in \operatorname{co}\left\{x^{1}, \ldots, x^{N}\right\}$ such that $\operatorname{supp}\left(\bar{x}^{1}\right)=\cup\left\{\operatorname{supp}\left(x^{i}\right) \mid i \in\{1, \ldots, N\}\right\}$. Moreover, the inductive hypotheses guarantees the existence of some $\bar{x} \in \operatorname{co}\left\{\bar{x}^{1}, x^{N+1}\right\}$ such that $\operatorname{supp}(\bar{x})=\operatorname{supp}\left(\bar{x}^{1}\right) \cup$ $\operatorname{supp}\left(x^{N+1}\right)$. We derive from the definition of $\bar{x}^{1}$ that $\operatorname{supp}(\bar{x})=\cup\left\{\operatorname{supp}\left(x^{i}\right) \mid i \in\{1, \ldots, N+1\}\right\}$. Also, $\bar{x}^{1} \in$ $\operatorname{co}\left\{x^{1}, \ldots, x^{N}\right\}$ and $\bar{x} \in \operatorname{co}\left\{\bar{x}^{1}, x^{N+1}\right\}$ imply that $\bar{x} \in \operatorname{co}\left\{x^{1}, \ldots, x^{N}, x^{N+1}\right\}$, which ends the proof.

Lemma A.9. Let $C \subset X$ be a convex nonempty set, and $J=\cup\{\operatorname{supp}(x) \mid x \in C\}$. If $J$ is finite, then there exists $\bar{x} \in C$ such that $\operatorname{supp}(\bar{x})=J$.

Proof. Since $J$ is finite, there exists a finite family $\left\{x^{1}, \ldots, x^{N}\right\} \subset C$ such that $J=\cup\left\{\operatorname{supp}\left(x^{i}\right) \mid i \in\{1, \ldots, N\}\right\}$. It suffices then to apply the previous lemma to obtain such $\bar{x} \in \operatorname{co}\left\{x^{1}, \ldots, x^{N}\right\} \subset C$.

Acknowledgements. This material is supported by the Center for Brains, Minds and Machines, funded by NSF STC award CCF-1231216, and the Air Force project FA9550-17-1-0390. G. Garrigos is supported by the European Research Council (ERC project NORIA), and part of his work was done while being a postdoc at the LCSL-IIT@MIT. L. Rosasco acknowledges the financial support of the Italian Ministry of Education, University and Research FIRB project RBFR12M3AC. S. Villa is supported by the INDAM GNAMPA research project 2017 Algoritmi di ottimizzazione ed equazioni di evoluzione ereditarie. L. Rosasco and S. Villa acknowledge the financial support from the EU project 777826 - NoMADS.

\section{REFERENCES}

[1] H. Attouch and J. Bolte, On the convergence of the proximal algorithm for nonsmooth functions involving analytic features. Math. Program. Ser. B 116 (2009) 5-16.

[2] H. Attouch, J. Bolte and B.F. Svaiter, Convergence of descent methods for semi-algebraic and tame problems: proximal algorithms, forward-backward splitting, and regularized Gauss-Seidel methods. Math. Program. 137 (2013) 91-129.

[3] A. Barbara, A. Jourani and S. Vaiter, Maximal Solutions of Sparse Analysis Regularization. Preprint arXiv:1703.00192 (2017).

[4] H.H. Bauschke and P. Combettes, Convex Analysis and Monotone Operator Theory, CMS Books in Mathematics, 2nd edn. Springer, Berlin (2017).

[5] A. Blanchet and J. Bolte, A family of functional inequalities: Lojasiewicz inequalities and displacement convex functions. J. Funct. Anal. 275 (2018) 1650-1673. 
[6] J. Bolte, T.-P. Nguyen, J. Peypouquet and B. Suter, From error bounds to the complexity of first-order descent methods for convex functions. Math. Programm. 165 (2017) 471-507.

[7] J.M. Borwein and A. Lewis, Partially finite convex programming, part I: Quasi relative interiors and duality theory. Math. Programm. 57 (1992) 15-48.

[8] K. Bredies and D.A. Lorenz, Linear convergence of iterative soft-thresholding. J. Fourier Anal. Appl. 14 (2008) $813-837$.

[9] P.L. Combettes and J.-C. Pesquet, Proximal thresholding algorithm for minimization over orthonormal bases. SIAM J. Optim. 18 (2007) 1351-1376.

[10] P.L. Combettes, S. Salzo and S. Villa, Consistency of regularized learning schemes in Banach spaces. Math. Programm. published online 2017-03-25.

[11] I. Daubechies, M. Defrise and C. De Mol, An iterative thresholding algorithm for linear inverse problems with a sparsity constraint. Commun. Pure Appl. Math. 57 (2004) 1413-1457.

[12] D. Davis and W. Yin, Convergence rate analysis of several splitting schemes, in Splitting Methods in Communication, Imaging, Science, and Engineering. Springer International Publishing, Switzerland (2016) 115-163.

[13] C. De Mol, E. De Vito and L. Rosasco, Elastic-net regularization in learning theory. J. Complexity 25 (2009) $201-230$.

[14] K. Degraux, G. Peyré, J. Fadili and L. Jacques, Sparse support recovery with non-smooth loss functions, in Advances in Neural Information Processing Systems (2016) 4269-4277.

[15] C. Dossal, A necessary and sufficient condition for exact recovery by 11 minimization. Comptes Rendus Mathématique 350 (2011) $117-120$.

[16] D. Drusvyatskiy and A.D. Lewis, Error bounds, quadratic growth, and linear convergence of proximal methods. Math. Oper. Res. (2018) published online.

[17] V. Duval and G. Peyré, Sparse spikes super-resolution on thin grids I: the LASSO. Inverse Probl. 33 (2017) 055008.

[18] J. Fadili, J. Malick and G. Peyré, Sensitivity analysis for Mirror-Stratifiable convex functions. Preprint arXiv:1707.03194 (2017).

[19] P. Frankel, G. Garrigos and J. Peypouquet, Splitting methods with variable metric for Kurdyka-Łojasiewicz functions and general convergence rates. J. Optim. Theory Appl. 165 (2015) 874-900.

[20] G. Garrigos, L. Rosasco and S. Villa, Convergence of the Forward-Backward algorithm: beyond the worst case with the help of geometry. Preprint arXiv:1703.09477 (2017).

[21] E.T. Hale, W. Yin and Y. Zhang, Fixed-point continuation for $\ell_{1}$-minimization: methodology and convergence. SIAM $J$. Optim. 19 (2008) 1107-1130.

[22] W.L. Hare and A.S. Lewis, Identifying active constraints via partial smoothness and prox-regularity. J. Convex Anal. 11 (2004) 251-266.

[23] J.-B. Hiriart-Urruty and C. Lemaréchal, Convex Analysis and Minimization Algorithms I: Fundamentals. Springer Science \& Business Media, Berlin (1993).

[24] B. Lemaire, Stability of the iteration method for non expansive mappings. Serdica Math. J. 22 (1996) 331-340.

[25] G. Li, Global error bounds for piecewise convex polynomials. Math. Programm. 137 (2013) 37-64.

[26] J. Liang, J. Fadili and G. Peyré, Local linear convergence of Forward-Backward under partial smoothness, in Advances in Neural Information Processing Systems (2014) 1970-1978.

[27] J. Liang, J. Fadili and G. Peyré, Activity identification and local linear convergence of Forward-Backward-type methods. SIAM J. Optim. 27 (2017) 408-437.

[28] S. Mosci, L. Rosasco, M. Santoro, A. Verri and S. Villa, Solving structured sparsity regularization with proximal methods, in Machine Learning and Knowledge Discovery in Databases, edited by J.L. Balcázar, F. Bonchi, A. Gionis, M. Sebag, ECML PKDD 2010. Vol 6322 of Lecture Notes in Computer Science. Springer, Berlin, Heidelberg (2010)

[29] I. Necoara, Y. Nesterov and F. Glineur, Linear convergence of first order methods for non-strongly convex optimization. Math. Programm. 175 (2018) 69-107.

[30] J. Nutini, M. Schmidt and W. Hare, "Active-set complexity" of proximal gradient: how long does it take to find the sparsity pattern?. Preprint arXiv:1712.03577 (2017).

[31] J. Peypouquet, Convex Optimization in Normed Spaces: Theory, Methods and Examples. Springer Science \& Buisness Media, Switzerland (2015).

[32] J. Peypouquet and S. Sorin, Evolution equations for maximal monotone operators: asymptotic analysis in continuous and discrete time. J. Convex Anal. 17 (2010) 1113-1163.

[33] R.T. Rockafellar, Convex Analysis, Vol. 28 of Princeton Mathematical Series. Princeton University Press, Princeton, NJ (1970).

[34] C. Zalinescu, Convex Analysis in General Vector Spaces. World Scientific, NJ (2002).

[35] H. Zou and T. Hastie, Regularization and variable selection via the elastic net. J. R. Stat. Soc. Ser. B 67 (2005) 301-320. 